\title{
The hydrodynamic force on a rigid particle undergoing arbitrary time-dependent motion at small Reynolds number $\dagger$
}

\author{
By PHILliP M. LOVALENTI AND JOHN F. BRADY \\ Department of Chemical Engineering, California Institute of Technology, Pasadena, \\ CA 91125 , USA
}

(Received 5 August 1992 and in revised form 27 May 1993)

The hydrodynamic force acting on a rigid spherical particle translating with arbitrary time-dependent motion in a time-dependent flowing fluid is calculated to $O(R e)$ for small but finite values of the Reynolds number, $R e$, based on the particle's slip velocity relative to the uniform flow. The corresponding expression for an arbitrarily shaped rigid particle is evaluated for the case when the timescale of variation of the particle's slip velocity is much greater than the diffusive scale, $a^{2} / v$, where $a$ is the characteristic particle dimension and $v$ is the kinematic viscosity of the fluid. It is found that the expression for the hydrodynamic force is not simply an additive combination of the results from unsteady Stokes flow and steady Oseen flow and that the temporal decay to steady state for small but finite $R e$ is always faster than the $t^{-\frac{1}{2}}$ behaviour of unsteady Stokes flow. For example, when the particle accelerates from rest the temporal approach to steady state scales as $t^{-2}$.

\section{Introduction}

This study is concerned with the unsteady motion of a rigid particle in an unbounded incompressible Newtonian fluid. The flow far from the particle may also be unsteady, but is taken to be uniform. In addition, we assume that the particle Reynolds number, $R e=U_{c} a / v$, based on a characteristic particle slip velocity, $U_{c}$, remains small throughout the particle motion. Here $a$ denotes the characteristic particle dimension and $v$ is the kinematic viscosity of the fluid. In particular, we solve for the nonsteady hydrodynamic force acting on the particle for small but finite values of the Reynolds number.

The motivation for studying such a problem at small Reynolds number is threefold. First, there is the basic question of what are the forces acting on a particle undergoing an arbitrary time-dependent motion. How do these forces depend on time and on the Reynolds number? Second, the problem has applications in particle dynamics. For example, in suspension mechanics Stokes flow often dominates the fluid motion, owing to small particle sizes and relatively large fluid viscosities. The inertia of the fluid and particle are then small corrections, and the present analysis represents a first step towards the inclusion of both convective and unsteady inertial effects in suspension mechanics. Third, there is considerable interest in the transport of small particles in turbulent flows (Reeks \& McKee 1984; Mei, Adrian \& Hanratty

$\uparrow$ With Appendix A by P. M. Lovalenti, J. F. Brady and Howard A. Stone; and Appendix D by E. J. Hinch 
1991; Young \& Hanratty 1991), and a large part of the unsteadiness arises from the turbulent velocity fluctuations. Determining unsteady hydrodynamic forces in these flows is necessary to determine particle motion, which has obvious implications for particle dispersion and deposition, particle-image velocimetry measurements (Adrian 1991), etc. In addition, through this problem we wish to demonstrate the relative simplicity of using the reciprocal theorem as an approach to computing inertial effects.

To determine the force correct to $O(R e)$, we do not solve for the detailed flow field; instead, we make use of the reciprocal theorem and the known results of steady (and, in some cases, unsteady) Stokes flow to avoid the detailed problem and proceed directly to computing the force. From the reciprocal theorem we obtain results for the hydrodynamic force in terms of inertial corrections to the steady Stokes drag. These corrections come from two sources. One is due to the unsteady nature of the flow and yields such contributions to the hydrodynamic force as the Basset force and the added mass. The other inertial corrections are due to weak convective effects and are the origin of the well-known $O(R e)$ Oseen correction to the steady Stokes drag. When both contributions are considered together, we shall see that the nature of the hydrodynamic force is determined by the characteristic timescale of the motion, $\tau_{c}$. When $\tau_{c}$ is small (e.g. $O\left(a^{2} / v\right)$ ), the unsteady inertial corrections dominate the hydrodynamic force. Only when $\tau_{c}$ becomes very large (i.e. $\tau_{c}>O\left(v / U_{c}^{2}\right)$ ) does the convective inertial correction take the form of the Oseen result. Further, and not surprisingly, we shall see that the two inertial contributions, unsteadiness and convection, are not simply additive. Indeed, the convective inertia changes fundamentally the temporal decay of the unsteady inertia from $t^{-\frac{1}{2}}$ to $t^{-2}$ when particles accelerate from rest, which has profound implications for the approach to steady state.

In the area of unsteady inertial effects, several results have been obtained by previous researchers. For example, Basset (1888) found the hydrodynamic force acting on a spherical particle undergoing arbitrary time-dependent motion in an otherwise quiescent fluid. The governing equations were the unsteady Stokes equations for a fluid of density $\rho$ and viscosity $\mu$ :

$$
-\nabla p+\mu \nabla^{2} u=\rho \frac{\partial u}{\partial t}, \quad \nabla \cdot u=0
$$

with boundary conditions

$$
\boldsymbol{u}=\boldsymbol{U}_{p}(t) \quad \text { when } \quad|x|=a ; \quad \boldsymbol{u}, p \rightarrow 0 \quad \text { as }|\boldsymbol{x}| \rightarrow \infty .
$$

Here, $u$ is the velocity of the fluid, $p$ the pressure, $U_{p}(t)$ the velocity of the particle, and $a$ its radius. Basset's result is

$$
F_{u s}^{H}(t)=-6 \pi \mu a U_{p}(t)-6 \pi \mu a\left(\frac{a^{2}}{\pi v}\right)^{\frac{1}{2}} \int_{-\infty}^{t} \frac{\dot{U}_{p}(s) \mathrm{d} s}{(t-s)^{\frac{1}{2}}}-\frac{2}{3} \pi \rho a^{3} \dot{U}_{p}(t),
$$

where $\dot{U}_{p}(t)$ is the acceleration of the particle. The first term is the pseudo-steady Stokes drag. The second is the Basset memory integral, which depends on the past history of the particle motion. It is a combination of both viscous and inertial contributions to the force in that it depends on both the viscosity of the fluid and the acceleration of the particle. The last term, a purely inertial contribution, is the so-called added mass. It represents the additional mass the particle appears to have due to the resistance to acceleration of the surrounding fluid.

Basset's result was extended to conditions where the flow far from the particle was other than uniform (Maxey \& Riley 1983) (see also Appendix A) and to particles 
of non-spherical shape (Lawrence \& Weinbaum 1986, 1988; Gavze 1990). An important result of the analysis by Lawrence \& Weinbaum (1988) is that the form of the hydrodynamic force becomes significantly more complicated than (1.2) for nonspherical particles. For example, they demonstrated the existence of distinct high- and low-frequency forms for the 'Basset' history force associated with the axisymmetric oscillation of a spheroid. Only for the special case of a sphere is the history force the same in the high- and low-frequency limits. Here we refer to the history force as that term proportional to the half-power of the frequency in the Fourier transform representation of the solution. In this way it retains the form of the second term of (1.2) in the time domain for arbitrary particle motion. We note also that Gavze (1990) recently presented a general theory for the force and torque acting on a particle of arbitrary shape undergoing rigid body motion in unsteady Stokes flow. Gavze derives expressions for the second-rank tensors that relate the force and torque to the particle's velocity and acceleration, and demonstrates their symmetry properties. The author identifies a steady resistance tensor associated with steady Stokes flow, a potential (or 'added mass') resistance tensor associated with potential flow, and a Basset resistance tensor that is a function of time.

One of the first treatments of convective inertia was the classic problem solved by Oseen $(1910,1913)$. He computed the first correction to the Stokes drag for small but finite values of the Reynolds number for a sphere held fixed in a steady uniform flow, $\boldsymbol{U}$. Oseen recognized that the governing equations near the particle were adequately described by the Stokes equations, but that far from the particle they were more appropriately given by the so-called Oseen equations:

$$
-\nabla p+\mu \nabla^{2} \boldsymbol{u}=\rho \boldsymbol{U} \cdot \boldsymbol{\nabla} \boldsymbol{u}, \quad \boldsymbol{\nabla} \cdot \boldsymbol{u}=0 .
$$

Oseen constructed a uniformly valid leading-order approximation to the flow field that satisfies (1.3) everywhere and obtained the hydrodynamic force expression

$$
F_{O s}^{H}=6 \pi \mu a U\left(1+\frac{3}{8} R e\right),
$$

where $\operatorname{Re}=a|\boldsymbol{U}| / v$. Although this result for the force is correct, Oseen did not compute the velocity field accurate to $O(R e)$. Proudman \& Pearson (1957) made use of singular perturbation theory to correctly compute the velocity field to this order, and extended Oseen's result to $O\left(R e^{2} \log (R e)\right)$. Brenner \& Cox (1963) generalized these results to particles of arbitrary shape.

Sano (1981) extended the Proudman \& Pearson result to the unsteady startup problem where $\boldsymbol{U}(t)=\boldsymbol{U} H(t)$ and the fluid is stationary everywhere for $t<0$. $(H(t)$ is the Heaviside function.) His solution for the hydrodynamic force is

$$
\begin{aligned}
F^{H}(t)= & 6 \pi \mu a U\left[H(t)+\frac{1}{3} \delta(t)+(\pi t)^{-\frac{1}{2}}+\frac{3}{8} \operatorname{Re}\left\{\left(1+\frac{4}{\operatorname{Re}^{4} t^{2}}\right) \operatorname{erf}\left(\frac{1}{2} \operatorname{Re}^{\frac{1}{2}}\right)\right.\right. \\
& \left.\left.+\frac{2}{(\pi t)^{\frac{1}{2}} \operatorname{Re}}\left(1-\frac{2}{\operatorname{Re}^{2} t}\right) \exp \left(-\frac{1}{4} \operatorname{Re}^{2} t\right)-\frac{8}{3(\pi t)^{\frac{1}{2}} \operatorname{Re}}\right\}+\frac{9}{40} \operatorname{Re} \ln \operatorname{Re}+O\left(\operatorname{Re}^{2}\right)\right] .
\end{aligned}
$$

Here, $t$ has been non-dimensionalized by $a^{2} / v, \delta(t)$ is the Dirac delta function, and $\operatorname{erf}(x)$ is the error function. The first three terms are the unsteady Stokes force, while the portion in curly brackets represents the unsteady Oseen contribution. This result was based on Bentwich \& Miloh's (1978) Laplace transform solution for the unsteady Oseen velocity field. It is Sano's result that most closely corresponds to the current 
work. We extend his result to conditions where the particle and the far-field flow can have general time dependence and to particles of arbitrary shape. Of particular interest in (1.5) is the fact that with the inclusion of the Oseen convective inertia the force approaches its steady value as $t^{-2}$ in contrast to the $t^{-\frac{1}{2}}$ predicted by the Basset term of (1.2) from just including the unsteady inertia. This much more rapid temporal decay has important implications for unsteady particle motion. Ockendon (1968) also found that on long timescales there is a change in the temporal decay due to the contribution from convective inertia. Recent numerical studies by Mei, Lawrence \& Adrian (1991) and Mei \& Adrian (1992) have also confirmed this change in temporal decay.

In order to appreciate the different physical processes occurring in unsteady particle motion, a consideration of the various timescales present is warranted. One can define a Strouhal number as $S l=\left(a / U_{c}\right) / \tau_{c}$, which is a measure of the timescale of variation, or unsteadiness, relative to the convective time $a / U_{c}$. For example, when a particle is accelerating from rest in stationary fluid, the appropriate timescale is $O\left(a^{2} / v\right)$, the time it takes vorticity to diffuse over the length $a$. In this case, $S l=O\left(R e^{-1}\right) \gg 1$, reflecting the fact that unsteady inertia is large compared to convective inertia. If one considers the problem of a particle released from rest and settling under gravity, initially the timescale is $O\left(a^{2} / v\right)$, but as the particle approaches its terminal velocity it progresses through an entire range of longer timescales. Thus, the Strouhal number initially would be very large but would become very small as steady state was being attained. In oscillatory motion $\tau_{c}=O\left(\omega^{-1}\right)$, where $\omega$ is the frequency of oscillation. Here again, the magnitude of $S l$ may take on very large or small values depending on the magnitude of $\omega$. Steady particle motion, on the other hand, corresponds to an infinite time scale of variation. Thus, for example, oscillatory particle motion coupled with steady motion provides a condition for the existence of duel timescales, one being $\omega^{-1}$ and the other infinite. Owing to the nonlinearity of the Navier-Stokes equations, these two effects cannot in general be separated into isolated contributions. Also, distinct magnitudes of $S l$ may not be identifiable at a given instant in time. Since we wish to consider arbitrary time-dependent motion, we must allow for the fact that the relative importance of unsteady and convective inertia, i.e. $S l$, may be of arbitrary magnitude and may change as a function of time. Thus, we shall explicitly leave the Strouhal number in the problem.

If we take as a prototypical example the problem of a spherical particle settling under gravity, our goal then is to compute the hydrodynamic force correct to $O(R e)$ as the particle accelerates from rest and approaches its terminal velocity. As we shall see, initially the hydrodynamic force is determined by unsteady Stokes flow, where the Stokes drag, Basset force, and the added mass are of equal importance. At this stage, vorticity has not diffused out to the Oseen distance of $O\left(a R e^{-1}\right)$, and, since there is no far-field Oseen region, one does not expect an $O(R e)$ Oseen-like correction to the drag. We find, for the case of a spherical particle, that on this timescale $\left(\tau_{c} \sim a^{2} / v\right)$ there is no convective inertial correction to the hydrodynamic force at $O(R e)$. (Under these conditions the convective inertial correction can be anticipated to enter at $O\left(R e^{2}\right)$.) In fact, not until $S l=O(R e)$, or $\tau_{c}=v / U_{c}{ }^{2}$, which corresponds to the time it takes vorticity to diffuse out to the Oseen distance, does the convective inertia of the fluid make a contribution to the hydrodynamic force at $O(R e)$. Finally, when the particle reaches its terminal velocity, the hydrodynamic force to $O(R e)$ is given by the Stokes drag plus the steady Oseen correction.

As remarked earlier, the Oseen convective inertia changes the approach to steady state from $t^{-\frac{1}{2}}$ to $t^{-2}$. To understand how this comes about we must first appreciate 
that the origin of the Basset, $t^{-\frac{1}{2}}$, scaling is due to the uniform, spherically symmetric diffusion of vorticity generated at the particle surface into regions of irrotational flow. At finite $R e$, however, this process does not continue indefinitely. Rather, when the vorticity has diffused out to the Oseen distance, it is swept up in the wake region behind the particle where it is transported by convection. As we shall show, this change in the mechanism of vorticity transport accounts for the change in the temporal behaviour of the hydrodynamic force. Because of this change in the temporal decay, a particle released from rest at finite Reynolds number will reach its terminal velocity much more rapidly than one would have predicted based on the Basset correction alone. This fact may explain why experimentalists have measured a steady terminal velocity when the length of their apparatus would not have permitted this if the Basset force was correct.

We can at this point estimate the temporal decay of the force from energy dissipation arguments and the known behaviour of unsteady Stokes flow and the wake region in Oseen flow. The rate of work done by the particle on the fluid is proportional to the volume integral over the fluid of the dissipation $\sigma: \nabla u$. As steady state is approached the temporal perturbation to the steady force is proportional to the 'dissipation deficit' - the dissipation due to the disturbance velocity - in the region of the fluid not yet reached by the transported vorticity. Vorticity is either confined to the vorticity diffusion volume for short timescales or to the truncated Oseen wake at finite Reynold numbers for long timescales. In both cases, in the region yet to be reached by the vorticity, at steady state the disturbance velocity $u$ will behave as $1 / r$ and the associated stress field $\sigma$ will be $1 / r^{2}$ from Stokes flow, where $r$ is the distance from the centre of the particle. When vorticity has not diffused out to the Oseen distance, $\left(\tau_{c} v\right)^{\frac{1}{2}}<v / U_{c}$, the velocity disturbance is confined to a spherical region of radius $(v t)^{\frac{1}{2}}$. Hence, the temporal correction to the force in dimensional form goes as

$$
F \sim \mu a^{2} U \int_{(v t)^{\frac{1}{2}}}^{\infty} r^{-2} r^{-2} \mathrm{~d} V \sim \mu a^{2} U(v t)^{-\frac{1}{2}} \sim \mu a U\left(a^{2} / v t\right)^{\frac{1}{2}}
$$

This is just the $t^{-\frac{1}{2}}$ approach to steady state of the Basset force.

However, when vorticity has diffused out to the Oseen distance, $(v t)^{\frac{1}{2}} \approx a R e^{-1}$, the velocity disturbance is gathered up by the convective flow and confined to the (unsteady) Oseen wake. Denoting by $z$ the distance behind the particle, the length of the wake grows convectively as $z \sim U t$, while its width $\left(A^{\frac{1}{2}}\right)$ grows diffusively as $(z v / U)^{\frac{1}{2}}=(v t)^{\frac{1}{2}}$, where $A$ is the cross-sectional area of the wake. Thus, letting $\sigma$ scale as $\nabla u$ and $|u| \sim 1 / r \approx 1 / z$ from Stokes flow, the temporal correction to the dimensional force goes as

$$
F \sim \mu a^{2} U \int z^{-2} z^{-2} \mathrm{~d} z \mathrm{~d} A \sim \mu a^{2} U z^{-3} A \sim \mu a^{2} U(U t)^{-3}(v t) \sim \mu a U(a U / v)\left(v / U^{2} t\right)^{2} ;
$$

the advertised $t^{-2}$ temporal decay. We shall see explicitly below by detailed analysis that this is indeed the proper temporal decay. In Appendix D, E. J. Hinch offers another manner in which to estimate the temporal decay through a consideration of the mass and momentum deficit in the wake.

In what follows, we obtain the form for the hydrodynamic force to $O(R e)$ that spans the transition from the unsteady Stokes force to the Oseen drag for arbitrary time-dependent motion. As expected from the above discussion and from the result of Sano (1981), it is found that the results are not simply additive. We shall also see that the form of the result will greatly simplify for the case of a spherical particle. 
In addition, we obtain a simplified expression for arbitrarily shaped particles for the case when the timescale is long $\left(\gg a^{2} / v\right)$. Under this condition, both unsteady and convective inertial corrections can be treated through singular perturbations to steady Stokes flow and will require only the steady Stokes velocity field associated with the translating particle.

In the next section, the full governing equations for the problem will be posed and the relevant dimensionless parameters identified. In $\$ 3$, the reciprocal theorem is presented for computing the inertial correction to the steady Stokes drag. A general derivation of the reciprocal theorem with inertial effects is given in Appendix A. This derivation also generalizes the work of Maxey \& Riley (1983). Since the inertial correction in the reciprocal theorem is expressed as a volume integral of functions of the velocity field over the entire fluid domain, we apply scaling arguments in $\$ 4$ to obtain a uniformly valid velocity field that is valid for all timescales. In $\S 5$, we combine this velocity field with the reciprocal theorem to obtain an expression for the hydrodynamic force correct to $O(R e)$. In the following three sections, calculations are performed to simplify the expression under various conditions of timescales and particle shape. Included in $\$ 7$ is the application of the expression to the numerical calculation of the settling velocity of a spherical particle released from rest. In the last section, we conclude with a discussion of the results.

\section{Governing equations and boundary conditions}

The governing equations for the problem are given by the full Navier-Stokes equations

$$
\begin{gathered}
-\nabla p^{\prime}+\mu \nabla^{2} \boldsymbol{u}^{\prime}=\rho\left(\frac{\partial \boldsymbol{u}^{\prime}}{\partial t}+\boldsymbol{u}^{\prime} \cdot \nabla \boldsymbol{u}^{\prime}\right), \\
\nabla \cdot \boldsymbol{u}^{\prime}=0,
\end{gathered}
$$

with the boundary conditions

$$
\begin{gathered}
\boldsymbol{u}^{\prime}=\boldsymbol{U}_{p}(t) \quad \text { on the surface of the particle, } \\
\boldsymbol{u}^{\prime} \rightarrow \boldsymbol{U}^{\infty}(t), p^{\prime} \rightarrow p^{\infty} \quad \text { as } \quad\left|\boldsymbol{x}-\boldsymbol{Y}_{p}(t)\right| \rightarrow \infty .
\end{gathered}
$$

The uniform undisturbed flow far from the particle is $U^{\infty}(t)$, and $\boldsymbol{Y}_{p}(t)$ is the position vector for the centre of mass of the particle. The pressure $p^{\infty}$ satisfies

$$
-\nabla p^{\infty}=\operatorname{ReSl} \dot{U}^{\infty}(t) \text {. }
$$

For our purposes, it is more convenient to pose the problem in a translating coordinate system with the origin at the instantaneous centre of the particle. Also, since we wish to deal with a velocity field that decays to zero far from the particle, we consider the disturbance velocity and pressure fields. Thus, letting $\boldsymbol{r}=\boldsymbol{x}-\boldsymbol{Y}_{p}(t)$, $\boldsymbol{u}=\boldsymbol{u}^{\prime}-\boldsymbol{U}^{\infty}(t)$ and $p=p^{\prime}-p^{\infty}$, the problem becomes in dimensionless form

$$
\begin{gathered}
-\nabla p+\nabla^{2} \boldsymbol{u}=\operatorname{ReSl} \frac{\partial \boldsymbol{u}}{\partial t}+\operatorname{Reu} \cdot \nabla \boldsymbol{u}-\operatorname{Re} \boldsymbol{U}_{s}(t) \cdot \nabla \boldsymbol{u}, \\
\boldsymbol{\nabla} \cdot \boldsymbol{u}=0,
\end{gathered}
$$

with

$$
\begin{gathered}
u=U_{s}(t) \quad \text { on the surface of the particle, } \\
\qquad u, p \rightarrow 0 \quad \text { as } \quad|r| \rightarrow \infty
\end{gathered}
$$


The slip velocity of the particle $U_{s}(t)$ is defined by $\boldsymbol{U}_{s}(t)=U_{p}(t)-U^{\infty}(t)$. The Reynolds number is $R e=U_{c} a / v$, and the Strouhal number is $S l=\left(a / U_{c}\right) / \tau_{c}$. Here, $a, U_{c}$, and $\tau_{c}$ are the characteristic particle dimension, particle slip velocity, and timescale, respectively. $U_{s}(t)$ and $\boldsymbol{u}$ have been non-dimensionalized by $U_{c}, \boldsymbol{r}$ by $a$, and $p$ by $\mu U_{c} / a$. We do not prescribe an initial condition, but only require that $\boldsymbol{u}$ remains bounded for all time, $-\infty<t<\infty$.

\section{The reciprocal theorem}

As stated in the introduction, we make use of the reciprocal theorem to compute the hydrodynamic force for the problem stated above. In Appendix A, we derive the reciprocal theorem for the total hydrodynamic force acting on a spherical particle undergoing the time-dependent motion $\boldsymbol{U}_{p}(t)$ in a time- and space-dependent flow, $\boldsymbol{v}^{\infty}(\boldsymbol{x}, t)$, and for the total hydrodynamic force on an arbitrarily shaped particle in a uniform time-dependent flow, $U^{\infty}(t)$. For a uniform flow $\boldsymbol{U}^{\infty}(t)$ about a particle of arbitrary shape with slip velocity $U_{s}(t)$, the reciprocal theorem takes the following form:

$$
\boldsymbol{F}^{H}(t)=\boldsymbol{F}_{s}^{H}(t)+\operatorname{ReSl} \widetilde{V}_{p} \dot{U}^{\infty}(t)-\int_{V_{f}} \boldsymbol{f}(\boldsymbol{u}) \cdot \boldsymbol{M}(\boldsymbol{r}) \mathrm{d} V,
$$

where the hydrodynamic force, $F^{H}(t)$, has been non-dimensionalized by $\mu a U_{c}$ and the volume of the particle, $\widetilde{V}_{p}$, by $a^{3}$. Here, $F_{s}^{H}(t)$ is the pseudo-steady Stokes drag on the particle under the given flow conditions associated with the problem; for example, for a sphere $F_{s}^{H}(t)=-6 \pi U_{s}(t)$. The function $f(u)$ is given by the right-hand side of $(2.3 a)$ and satisfies the following series of equalities:

$$
\begin{aligned}
\boldsymbol{f}(\boldsymbol{u}) & =\operatorname{ReSl} \frac{\partial \boldsymbol{u}}{\partial t}+\operatorname{Re}\left\{\boldsymbol{u} \cdot \nabla \boldsymbol{u}-\boldsymbol{U}_{s}(t) \cdot \nabla \boldsymbol{u}\right\} \\
& =\nabla \cdot \boldsymbol{\sigma} \\
& =-\nabla p+\nabla^{2} \boldsymbol{u},
\end{aligned}
$$

where $\sigma$ and $\boldsymbol{u}$ are, respectively, the stress and velocity fields associated with the full disturbance Navier-Stokes problem given by (2.3). The inertia of the fluid is represented by $\boldsymbol{f}$ in the sense that $\boldsymbol{f}=0$ under steady Stokes flow conditions when $R e \equiv 0$.

The second-order tensor $\boldsymbol{M}(\boldsymbol{r})$ in (3.1) is defined by the condition that $\boldsymbol{M}(\boldsymbol{r}) \cdot \boldsymbol{U}_{\boldsymbol{p}}$ is the Stokes velocity field for the particle translating with constant velocity $U_{p}$ in a stationary fluid. For example, for a spherical particle $\boldsymbol{M}(\boldsymbol{r})$ is given by

$$
M(r)=\frac{3}{4}\left(\frac{1}{r}+\frac{r r}{r^{3}}\right)+\frac{3}{4}\left(\frac{1}{3 r^{3}}-\frac{r r}{r^{5}}\right),
$$

where $r=|r|$ and $\boldsymbol{l}$ is the idem tensor. We note that the centre of the coordinate system is at the particle centre and that the volume integral in the reciprocal theorem is over the entire volume of fluid.

As an added note, the reciprocal theorem is an exact result; if $\boldsymbol{u}$ is known, (3.1) yields the exact hydrodynamic force acting on the particle for arbitrary values of the Reynolds number. This is in contrast to a similar analysis presented by Leal (1980) for bounded domains which were limited to regular perturbations in small Re. By formulating the disturbance problem, we are able to remove this limitation and treat the general problem. 
Our goal now is to approximate the volume integral of (3.1) to give the inertial contribution to the hydrodynamic force correct to $O(R e)$ valid for all times. However, since the volume integral in (3.1) is over the entire fluid domain, the approximation needed must be uniformly valid over this region. It is well known that the solution to Stokes equations do not provide a valid description far from the particle, and singular perturbations will be necessary to construct a uniformly valid approximation.

In order to further motivate the necessity of singular perturbation theory, consider the result of substituting the steady Stokes velocity field for a spherical particle (the solution to (2.3) with $R e \equiv 0$ ) into the reciprocal theorem through the expression for $\boldsymbol{f}(\boldsymbol{u})$ given by $(3.2 a)$. This velocity field decays as $1 / r$ far from the particle, which is also true of the $\boldsymbol{M}$-field, as can be seen from (3.3). Thus, the integrand associated with the first term of $f, \operatorname{ReSl} \partial u / \partial t \cdot M$, decays as $1 / r^{2}$. (Note that the integrand in this case can be expressed as $\operatorname{ReSi} \boldsymbol{M} \cdot \boldsymbol{M} \cdot \dot{\boldsymbol{U}}_{s}(t)$.) This yields a divergent contribution to the volume integral of the reciprocal theorem. The contribution from the third term in $f$ is conditionally convergent, being $1 / r^{3}$.

The next obvious choice would be to consider the unsteady Stokes velocity field since it decays as $O\left(1 / r^{3}\right)$ and would eliminate the convergence difficulties. As it turns out, however, for the case of a sphere this only yields the unsteady inertial corrections associated with the last two terms of (1.2) and fails to produce convective inertial corrections due to the symmetry of the unsteady Stokes field. Since the unsteady Stokes flow must be linear in $U_{s}(t)$, it must be an even function of $r$, as $r$ is the only other vector in the problem. Since $f$ is then odd in $r$ and $M(r)$ even, the integrand is odd in $\boldsymbol{r}$ and the volume integral is zero upon angular integration. Clearly, more detailed considerations of the appropriate 'approximate' velocity field are necessary in order to correctly include the convective inertial contributions to $O(R e)$. (Note that although for non-spherical particles convective inertial contributions will be produced by these terms with the unsteady Stokes velocity field, it will not be the complete correction.)

\section{The uniformly valid velocity field}

We see from the governing equation (2.3) that the parameters that determine the form of the flow field are the Reynolds number and the Strouhal number (or, more appropriately, $R e$ and the product $R e S l$ ). The Reynolds number indicates the magnitude of the convective inertia relative to viscous forces, while $R e S l$ is a measure of the relative magnitude of the unsteady inertia of the fluid. Throughout the analysis that follows we assume that $R e \ll 1$. As we have seen, however, the Strouhal number may take on a range of magnitudes depending on the timescale of variation, and, since we do not restrict the timescale, $R e S l$ may also range in magnitude. In Appendix B, a formal perturbation analysis is used to develop scaling arguments for constructing a velocity field which is uniformly valid in space and time. Here we simply outline the results and present the reasoning used to obtain the uniformly valid field.

The most important point one finds from the scaling analysis is that singular perturbations are not required for particle motions with timescales smaller than $v / U_{c}^{2}$, i.e. such that $R e S l \gg R e^{2}$. With these conditions the unsteady Stokes velocity field, which we denote as $\boldsymbol{v}_{0}$, is uniformly valid in the entire fluid domain to leading order. There is no Oseen region where the convective inertia is of the same order as unsteady inertia or viscous forces. This feature of the flow is simply a result of the fact that the vorticity generated by such motion cannot diffuse out to the Oseen distance, $a R e^{-1}$. 
For particle motions with longer timescales, such that $R e S l \leqslant R e^{2}$, singular perturbations are required in order to take into account the Oseen region. The basic idea in constructing a uniformly valid field through singular perturbation techniques is to add the inner field, valid to the appropriate level of accuracy in the inner region, to the outer field and subtract the parts they have in common in their region of overlap. In the present case, we identify the inner region as that within the Oseen distance, and the outer region as that outside the Oseen distance. The leading-order field in the inner region, valid for all timescales, satisfies the unsteady Stokes equations. In the outer region the leading-order velocity field is given by the point-forced unsteady Oseen equations (see (B 8)). Only the point-forced equations are required to leading order because the dominant disturbance produced by the particle at large distances is that due to the force monopole. The velocity field that is common to both is that resulting from the point-forced unsteady Stokes equations (see (B 4)), and this field must be subtracted to prevent a double-counting of the contributions from the inner and outer regions. We denote the outer field less its overlap with the inner region as $\operatorname{Rev}_{1}^{p+}$.

Although, strictly speaking, the leading-order inner field on these long time scales is the steady Stokes velocity field, by replacing it with the unsteady Stokes field we have actually constructed a field uniformly valid in space and time. This is a result of the fact that the unsteady Stokes field on long timescales, $\tau_{c} \geqslant v / U_{c}^{2}$, will reduce to leading order to the steady Stokes field in the inner region and to the point-forced unsteady Stokes field in the outer region, the behaviour of which is removed by the common part field discussed above. On the other hand, on short timescales, $\tau_{c}<v / U_{c}^{2}$, the 'outer' unsteady Oseen field will reduce to the point-forced unsteady Stokes field to leading order, which similarly will be removed by the common part field. In both cases we obtain the desired velocity field to leading order: a steady Stokes inner field plus unsteady Oseen outer field for long timescales, and a uniformly valid unsteady Stokes field for short timescales.

An additional level of accuracy to the unsteady Stokes field is required for particle motions with timescales of $a^{2} / v$ or smaller, or $\operatorname{ReSl} \geqslant O(1)$. Under these conditions, the regular perturbation to the unsteady Stokes field for convective inertia (see (B 2)) is also required in general to obtain the proper $O(R e)$ correction to the unsteady term, $\operatorname{ReSl} \partial u / \partial t$, in (3.2a). This field is denoted $\operatorname{Rev}_{1}$. However, one must be careful in adding this field to prevent double-counting contributions already included by $R e v_{1}^{p+}$. Their common part, the point-forced portion of the regular perturbation field denoted by $\operatorname{Re} v_{1}^{p}$, must be subtracted. Having taken these requirements into account, the final expression for the uniformly valid velocity field for all timescales is

$$
\boldsymbol{u}^{u v}=\boldsymbol{v}_{0}+\operatorname{Rev}_{1}^{p+}+\operatorname{Re} \boldsymbol{v}_{1}-\operatorname{Rev}_{1}^{p} .
$$

(As a reminder from Appendix B, a subscript 0 indicates a leading-order field and a 1 indicates a velocity field contribution due solely to convective inertia, a superscript $p$ signifies a point-forced field, and the superscript + indicates that convective inertial terms are retained to leading order in the governing equation.) The $\boldsymbol{v}_{0}$-field is the unsteady Stokes velocity field and $R e v_{1}$ is its regular perturbation for convective inertia. The $R e v_{1}^{p+}$-field is the point-forced unsteady Oseen field with the point-forced unsteady Stokes field subtracted off. It represents a singularly perturbed correction for convective inertia, and its governing equation is given by (B 18). The last term, $R e v_{1}^{p}$, is necessary to prevent a double-counting of contributions from $\operatorname{Re} \boldsymbol{v}_{1}$ and $R e \boldsymbol{v}_{1}^{p+}$, and its governing equation is given by (B 15).

At this point one can derive an additional result from the scaling analysis of 
Appendix B. Although it is true that when $\tau_{c} \ll v / U_{c}^{2}$ there is no region in the fluid domain where convective inertia is of the same magnitude as unsteady inertia, the converse of this condition is not true. That is, when $\tau_{c} \gg v / U_{c}^{2}$ there will always be a far-field region where convective and unsteady inertia (as well as viscous forces) are of equal importance. This region is a result of the finite length of the Oseen wake for finite times. One can predict the temporal decay given at the end of $\$ 1$ from the scalings for this wake region given by (B 11) when $R e S l \ll R e^{2}$. That is, if we use the (B11) scalings in the full integral portion of the reciprocal theorem (3.1) we find

$$
\begin{aligned}
\boldsymbol{f}(\boldsymbol{u}) & \sim O\left(S l^{3}\right), \\
\mathbf{M} & \sim O(S l), \\
\mathrm{d} V=\mathrm{d} x \mathrm{~d} y \mathrm{~d} z & \sim O\left(\left(\operatorname{ReSl^{2})^{-1}),}\right.\right.
\end{aligned}
$$

which when these are combined yield a contribution to the hydrodynamic force that is $O\left(S l^{2} / R e\right)$.

\section{Expression for the hydrodynamic force to $O(R e)$}

With the above description of an approximation for the velocity field uniformly valid in space and time, we now use the reciprocal theorem (3.1) to obtain an expression for the hydrodynamic force, correct to $O(R e)$, which is uniformly valid in time. To evaluate the inertial contributions to the force, we need to approximate the volume integral of (3.1). This calculation is performed by combining the uniformly valid velocity field (4.1) with the first expression for $\boldsymbol{f}(\boldsymbol{u})$ given in $(3.2 a)$ :

$$
\begin{aligned}
f(u) \sim f\left(u^{u v}\right)= & \operatorname{ReSl} \frac{\partial v_{0}}{\partial t}+\operatorname{Re}\left(\operatorname{ReSl} \frac{\partial v_{1}}{\partial t}+v_{0} \cdot \nabla v_{0}-U_{s}(t) \cdot \nabla v_{0}\right) \\
& +\operatorname{ReSl}\left(\operatorname{Re} \frac{\partial v_{1}^{p+}}{\partial t}-\operatorname{Re} \frac{\partial v_{1}^{p}}{\partial t}\right)-\operatorname{Re}^{2} U_{s}(t) \cdot \nabla v_{1}^{p+}+\ldots .
\end{aligned}
$$

The first four terms on the right-hand side of (5.1) represent contributions from the unsteady Stokes equations and its regular $O(R e)$ perturbation for convection. The additional terms shown only make important contributions in the outer "Oseen" region and only when $R e S l \leqslant O\left(\operatorname{Re}^{2}\right)$. As we shall see in what follows, they are necessary to get the correct force to $O(R e)$. Scaling arguments can be used to show that the terms not shown combine (as in $\operatorname{Re}^{2} U_{s}(t) \cdot \nabla\left(v_{1}-v_{1}^{p}\right)$ ) to make contributions to the hydrodynamic force smaller than $O(R e)$ for all time and can therefore be neglected.

When this approximation to $\boldsymbol{f}(\boldsymbol{u})$ is combined with the reciprocal theorem (3.1), we obtain

$$
\begin{aligned}
\boldsymbol{F}^{H}(t)= & \boldsymbol{F}_{s}^{H}(t)+\operatorname{ReSl} \widetilde{V}_{p} \dot{U}^{\infty}(t)-\int_{V_{f}} \operatorname{ReSl} \frac{\partial \boldsymbol{v}_{0}}{\partial t} \cdot \boldsymbol{M} \mathrm{d} V \\
& -\operatorname{Re} \int_{V_{f}}\left(\operatorname{ReSl} \frac{\partial \boldsymbol{v}_{1}}{\partial t}+\boldsymbol{v}_{0} \cdot \nabla \boldsymbol{v}_{0}-\boldsymbol{U}_{s}(t) \cdot \nabla \boldsymbol{v}_{0}\right) \cdot \boldsymbol{M} \mathrm{d} V \\
& -\operatorname{Re} \int_{V_{f}}\left[\operatorname{ReSl}\left(\frac{\partial \boldsymbol{v}_{1}^{p+}}{\partial t}-\frac{\partial \boldsymbol{v}_{1}^{p}}{\partial t}\right)-\operatorname{Re} U_{s}(t) \cdot \nabla \boldsymbol{v}_{1}^{p+}\right] \cdot \boldsymbol{M} \mathrm{d} V+o(\operatorname{Re}) .
\end{aligned}
$$

The first integral yields precisely the hydrodynamic force due to the unsteady Stokes velocity field, $\boldsymbol{v}_{0}$, less the pseudo-steady Stokes drag, $\boldsymbol{F}_{s}^{H}(t)$. This is because the 
reciprocal theorem is an exact result for any inertial velocity field, including the unsteady Stokes field. Indeed, the result that this first integral represents the unsteady inertial contributions to the unsteady Stokes drag was verified by direct calculation using the known solution to the unsteady Stokes equations for a spherical particle. The second integral represents the contribution from the regular perturbation to the unsteady Stokes problem. The last integral is the contribution from the outer, singularly perturbed, velocity field. The entire point of the scaling analysis in $\$ 4$ was to obtain this last term. At large lengthscales and long times this volume integral is necessary to obtain the correct $O(R e)$ force, which can be seen by noting that the terms in square brackets in (5.2) are $O\left(R e^{2}\right)$ in the outer region when $S l=R e$ and that $M \mathrm{~d} V$ is $O\left(R e^{-2}\right)$ in this outer region (distances of $O\left(R e^{-1}\right)$ ), so that the volume integral itself is $O(1)$. Finally, we note that all integrals in (5.2) are absolutely convergent in space for all time. It is important to note that had we used the steady Stokes velocity field, $\boldsymbol{u}_{0}$, in place of the unsteady Stokes field, $\boldsymbol{v}_{0}$, in the integrals above, which would be a natural thing to do with the reciprocal theorem, the integrals would be conditionally convergent because the term $U_{s}(t) \cdot \nabla u_{0} \cdot M$, for example, is $O\left(1 / r^{3}\right)$. Using the unsteady Stokes solution removes this difficulty.

In order to greatly ease later calculations, we make a few simplifications of the last integral in (5.2). First, we note that because the contribution to the integral from the inner region (i.e. for distances smaller than $O(R e)$ ) is of lower order than $O(R e)$, the precise form of $\boldsymbol{M}$ in the inner region is not required. Only the far-field form of $\boldsymbol{M}$, which is to leading order given by the Stokeslet field, $\boldsymbol{M}_{p}$, is required. This field is the solution to

$$
-\nabla \boldsymbol{P}+\nabla^{2} \boldsymbol{M}_{p}=-6 \pi \Phi \delta(\boldsymbol{r}), \quad \nabla \cdot \boldsymbol{M}_{p}=0 .
$$

Here, $\boldsymbol{P}$ is a vector and $6 \pi \Phi$ is the Stokes resistance tensor associated with the particle: $-6 \pi \Phi \cdot U_{p}$ is the hydrodynamic force acting on the particle translating with velocity $U_{p}$. For a spherical particle, $\boldsymbol{M}_{p}$ is given by the first term of (3.3).

Next, we note that if we replace $\boldsymbol{M}$ with $\boldsymbol{M}_{p}$ we may extend the integration volume to include the volume of the particle. This extension may be performed because the $v_{1}^{p+}$ - and the $v_{1}^{p}$-fields are non-singular at the origin (i.e. the point force at the origin has been subtracted). Thus, the entire integrand behave at worst as $O\left(r^{-2}\right)$ as $r \rightarrow 0$, which is integrable. Also, upon integrating over the particle volume we make an error smaller than $O(R e)$, because the integrand is smaller than $O(R e)$ and the volume of integration is $O(1)$. Note that the difference field $v_{1}^{p+}-v_{1}^{p}$ is $O(R e)$ in this region.

The final simplification is in neglecting the term involving $R e v_{1}^{p}$. This can be done because the $v_{1}^{p}$ field is antisymmetric (or odd); that is,

$$
\boldsymbol{v}_{1}^{p}(-\boldsymbol{r})=-\boldsymbol{v}_{1}^{p}(\boldsymbol{r})
$$

as can be seen from the governing equations, (B 4) and (B 15). Thus, since the $\boldsymbol{M}_{\boldsymbol{p}}$-field is symmetric, that part of integrand involving the $\boldsymbol{v}_{1}^{p}$-field will be strictly antisymmetric and will angularly average to zero when integrated over any spherical surface.

With the above considerations taken into account, the expression to $O(R e)$ for the hydrodynamic force acting on a particle of arbitrary shape translating with a 
time-dependent velocity $\boldsymbol{U}_{s}(t)$ relative to a uniform stream, $\boldsymbol{U}^{\infty}(t)$, is

$$
\begin{aligned}
\boldsymbol{F}^{H}(t)= & \boldsymbol{F}_{u s}^{H}(t)+\operatorname{ReSl} \widetilde{V}_{p} \dot{U}^{\infty}(t) \\
& -\operatorname{Re} \int_{V_{f}}\left(\operatorname{ReSl} \frac{\partial \boldsymbol{v}_{1}}{\partial t}+\boldsymbol{v}_{0} \cdot \nabla v_{0}-U_{s}(t) \cdot \nabla v_{0}\right) \cdot \boldsymbol{M} \mathrm{d} V \\
& -\operatorname{Re} \int_{V_{\infty}}\left(\operatorname{ReSl} \frac{\partial \boldsymbol{v}_{1}^{p+}}{\partial t}-\operatorname{Re} U_{s}(t) \cdot \nabla v_{1}^{p+}\right) \cdot \boldsymbol{M}_{p} \mathrm{~d} V+o(\operatorname{Re}),
\end{aligned}
$$

where $V_{\infty}$ represents the entire volume of space. $F_{u s}^{H}(t)$ is that portion of the hydrodynamic force due solely to the unsteady Stokes problem (cf. (B 1)); and, with $\boldsymbol{U}_{s}(t)$ replacing $U_{p}(t)$, it becomes (1.2) for a spherical particle. The second integral is only required if $R e S l \leqslant O\left(R e^{2}\right)$; that is, this $O(R e)$ contribution comes from integration over the outer region when vorticity has diffused out to an $O\left(a R e^{-1}\right)$ distance. In the next section we evaluate this outer contribution. The contribution from unsteady Stokes flow and the regular perturbation (the first integral) will be commented on, and simplified for certain cases, in $\$ \$ 7$ and 8 .

\section{Calculation of the unsteady Oseen correction}

We write the contribution to the hydrodynamic force from the outer velocity field (the second integral in (5.5)) as

$$
\boldsymbol{F}_{\text {out }}^{H}(t)=-\int_{V_{\infty}} \boldsymbol{f}_{\text {out }}\left(\operatorname{Rev}_{1}^{p+}\right) \cdot \boldsymbol{M}_{p} \mathrm{~d} V,
$$

where

$$
f_{\text {out }}\left(\operatorname{Rev} v_{1}^{p+}\right)=\operatorname{Re}\left(\operatorname{ReSl} \frac{\partial v_{1}^{p+}}{\partial t}-\operatorname{Re} U_{s}(t) \cdot \nabla v_{1}^{p+}\right) .
$$

To evaluate this integral we make use of the convolution theorem and Fourier transforms to obtain

$$
F_{\text {out }}^{H}(t)=-\int_{V_{\infty}} \widehat{\boldsymbol{f}_{\text {out }}}\left(\operatorname{Rev} v_{1}^{p+}\right)(\boldsymbol{k}) \cdot \widehat{\boldsymbol{M}_{p}}(-\boldsymbol{k}) \mathrm{d} \boldsymbol{k},
$$

where ${ }^{\wedge}$ indicates the Fourier transform defined by

$$
\widehat{g}(\boldsymbol{k})=\int_{V_{\infty}} g(\boldsymbol{r}) \mathrm{e}^{-2 \pi i k \cdot r} \mathrm{~d} \boldsymbol{r}
$$

The Stokeslet field $\widehat{M}_{p}$ can be found from the Fourier transform of the governing equations (5.3):

$$
\widehat{M_{p}}=\frac{3}{2 \pi k^{2}}(I-n n) \cdot \Phi .
$$

Here, $\boldsymbol{n}$ is the spherical unit normal in $\boldsymbol{k}$-space (i.e. $\boldsymbol{n}=\boldsymbol{k} / \boldsymbol{k}$ ) and $k=|\boldsymbol{k}|$. The Fourier transform of $\boldsymbol{f}_{\text {out }}$ is found from the transform of (B 18) with (B 4) (see Appendix C). 
The result is

$$
\begin{aligned}
& \widehat{f_{\text {out }}}\left(\operatorname{Rev} v_{1}^{p+}\right)=\int_{-\infty}^{t} \frac{2 \pi \mathrm{i} R e}{\operatorname{ReSl}} \boldsymbol{k} \cdot\left(\boldsymbol{U}_{s}(s) \mathrm{e}^{2 \pi \mathrm{iRe}\left(\boldsymbol{Y}_{s}(t)-\boldsymbol{Y}_{s}(s)\right) \cdot k / R e S l}-U_{s}(t)\right) \\
& \times \mathrm{e}^{-4 \pi^{2} k^{2}(t-s) / R e s l} \boldsymbol{F}_{s}^{H}(s) \cdot(\boldsymbol{I}-\boldsymbol{n n}) \mathrm{d} s \\
& +\int_{-\infty}^{t}\left[1-\mathrm{e}^{2 \pi i \operatorname{Re}\left(Y_{\mathrm{s}}(t)-Y_{\mathrm{f}}(s)\right) \cdot \boldsymbol{k} / \operatorname{ReSt}}\right] \\
& \times \mathrm{e}^{-4 \pi^{2} k^{2}(t-s) / R e S l} \dot{\boldsymbol{F}}_{s}^{H}(s) \cdot(\boldsymbol{I}-\boldsymbol{n} \boldsymbol{n}) \mathrm{d} s,
\end{aligned}
$$

where $\boldsymbol{Y}_{s}(t)-\boldsymbol{Y}_{s}(s)$ is the integrated displacement of the particle relative to the fluid from time $s$ to the current time $t$, and $\boldsymbol{F}_{s}^{H}(s)$ is the pseudo-steady Stokes drag at time $s$. Also, it should be noted that in this equation, and in the equations that follow, $\boldsymbol{Y}_{s}(t)$ has been non-dimensionalized by $a S l^{-1}\left(=\tau_{c} U_{c}\right)$, not $a$. Since only the symmetric part (which is also the real part) of (6.5) can contribute to the volume integral of $(6.1 a)$, we write

$$
\begin{aligned}
& \widehat{\boldsymbol{f}_{\text {out }}}\left(\operatorname{Re} \boldsymbol{v}_{1}^{p+}\right)_{\text {sym }}=\int_{-\infty}^{t} \frac{-2 \pi R e}{\operatorname{ReSl}} \boldsymbol{k} \cdot \boldsymbol{U}_{s}(s) \sin \left(2 \pi \operatorname{Re}\left(\boldsymbol{Y}_{s}(t)-\boldsymbol{Y}_{s}(s)\right) \cdot \boldsymbol{k} / \operatorname{ReSl}\right) \\
& \times \mathrm{e}^{-4 \pi^{2} k^{2}(t-s) / R e S l} \boldsymbol{F}_{s}^{H}(s) \cdot(\boldsymbol{I}-\boldsymbol{n n}) \mathrm{d} s \\
& +\int_{-\infty}^{t}\left[1-\cos \left(2 \pi \operatorname{Re}\left(\boldsymbol{Y}_{s}(t)-\boldsymbol{Y}_{s}(s)\right) \cdot \boldsymbol{k} / \operatorname{ReSl}\right)\right] \\
& \times \mathrm{e}^{-4 \pi^{2} k^{2}(t-s) / R e s l} \dot{\boldsymbol{F}}_{s}^{H}(s) \cdot(\boldsymbol{I}-\boldsymbol{n} \boldsymbol{n}) \mathrm{d} s .
\end{aligned}
$$

If we combine (6.4) and (6.6) in (6.2) and perform the $k$-integration, the result is

$$
\begin{aligned}
\boldsymbol{F}_{\text {out }}^{H}(t)= & \frac{3 R e^{2}}{16 \pi^{\frac{3}{2}}(\operatorname{ReSl})^{\frac{1}{2}}} \int_{-\infty}^{t} \frac{\boldsymbol{F}_{s}^{H}(s)}{(t-s)^{\frac{1}{2}}} \boldsymbol{U}_{s}(s)\left(\frac{\boldsymbol{Y}_{s}(t)-\boldsymbol{Y}_{s}(s)}{t-s}\right) \vdots \boldsymbol{G}_{1}(t, s) \mathrm{d} s \cdot \Phi \\
& -\frac{3(\operatorname{ReSl})^{\frac{1}{2}}}{8 \pi^{\frac{3}{2}}} \int_{-\infty}^{t} \frac{\dot{\boldsymbol{F}}_{s}^{H}(s)}{(t-s)^{\frac{1}{2}}} \cdot \boldsymbol{G}_{2}(t, s) \mathrm{d} s \cdot \Phi,
\end{aligned}
$$

where

$$
\begin{gathered}
\mathbf{G}_{1}(t, s)=\int_{\Omega} n \boldsymbol{n}(I-n n) \mathrm{e}^{-(A(t, s) \cdot n)^{2}} \mathrm{~d} \Omega \\
\mathbf{G}_{2}(t, s)=\int_{\Omega}(I-n \boldsymbol{n})\left(1-\mathrm{e}^{-(A(t, s) \cdot n)^{2}}\right) \mathrm{d} \Omega,
\end{gathered}
$$

and

$$
A(t, s)=\frac{R e}{2}\left(\frac{t-s}{\operatorname{ReSl}}\right)^{\frac{1}{2}}\left(\frac{\boldsymbol{Y}_{s}(t)-\boldsymbol{Y}_{s}(s)}{t-s}\right) ;
$$

here $\Omega$ represents the angular integration over a spherical surface.

Now if we consider the situation with $\dot{U}_{s}(t)=0$ we can obtain the steady-state result for $\boldsymbol{F}_{\text {out }}^{H}$ :

$$
\boldsymbol{F}_{\text {out }}^{H}=-6 \pi\left(\frac{3}{8} \operatorname{Re}\left[\frac{3}{2}(\Phi \cdot p) \cdot \Phi-\frac{1}{2}(\Phi \cdot p) \cdot p \boldsymbol{p} \cdot \Phi\right]\right)\left|U_{s}\right|^{2},
$$


where

$$
\boldsymbol{p}=\frac{\boldsymbol{U}_{s}}{\left|\boldsymbol{U}_{s}\right|}
$$

Here we have used the fact that

$$
F_{s}^{H}=-6 \pi \Phi \cdot U_{s}
$$

This result agrees with the Oseen correction to the Stokes drag obtained by Brenner (1961) for an arbitrary particle shape.

In what follows we present some simplified expressions for the unsteady Oseen correction to the Stokes drag. The unsteady Oseen correction will be defined by

$$
F_{O s c}^{H}(t)=F_{o u t}^{H}(t)+\left(\frac{R e S l}{\pi}\right)^{\frac{1}{2}} \int_{-\infty}^{t} \frac{\dot{F}_{s}^{H}(s)}{(t-s)^{\frac{1}{2}}} \mathrm{~d} s \cdot \Phi .
$$

The first term on the right-hand side is given by (6.7). The second term represents the long-time expression of the "Basset" history force for an arbitrarily shaped particle and, as will be shown in $\S 8$, is the dominant long-time temporal contribution from unsteady Stokes flow. This definition is chosen because we wish to show the long-time asymptotic behaviour for various particle slip velocities. In all cases, we find that the long-time tail decays faster than the $t^{-\frac{1}{2}}$ predicted by unsteady Stokes flow.

First note that the second integral in the expression for $F_{\text {out }}^{H}(t),(6.7 a)$, may be written as

$$
\left[\frac{3(\operatorname{Re} S l)^{\frac{1}{2}}}{8 \pi^{\frac{3}{2}}} \int_{-\infty}^{t} \frac{\dot{\boldsymbol{F}}_{s}^{H}(s)}{(t-s)^{\frac{1}{2}}} \cdot \int_{\Omega}(I-n \boldsymbol{n}) \mathrm{e}^{-(A \cdot n)^{2}} \mathrm{~d} \Omega \mathrm{d} s-\left(\frac{\operatorname{ReSl}}{\pi}\right)^{\frac{1}{2}} \int_{-\infty}^{t} \frac{\dot{\boldsymbol{F}}_{s}^{H}(s)}{(t-s)^{\frac{1}{2}}} \mathrm{~d} s\right] \cdot \Phi,
$$

where it is seen that the second term cancels identically with the long-time 'Basset' history force term in (6.10). Next, performing integration by parts in $s$ on the first integral in (6.11) and combining with the first integral of $(6.7 a)$, we obtain the following result for $F_{o s c}^{H}(t)$ :

$$
\begin{aligned}
\boldsymbol{F}_{\text {Osc }}^{H}(t)=\frac{3}{8} \frac{(\operatorname{Re} S l)}{\pi^{\frac{3}{2}}}\left\{\int _ { - \infty } ^ { t } \int _ { \Omega } \left[\left\{\boldsymbol{F}_{s}^{H}(s)(\boldsymbol{A} \cdot \boldsymbol{n})^{2} \mathrm{e}^{-(\boldsymbol{A} \cdot \boldsymbol{n})^{2}}\right.\right.\right. \\
\left.\left.\left.+\frac{1}{2}\left(\boldsymbol{F}_{s}^{H}(t)-\boldsymbol{F}_{\mathrm{s}}^{H}(s) \mathrm{e}^{-(\boldsymbol{A} \cdot \boldsymbol{n})^{2}}\right)\right\} \cdot \frac{(\boldsymbol{I}-\boldsymbol{n} \boldsymbol{n})}{(t-s)^{\frac{3}{2}}}\right] \mathrm{~d} s \mathrm{~d} \Omega\right\} \cdot \Phi .
\end{aligned}
$$

Further simplification of the angular integration can be achieved by dividing $\boldsymbol{F}_{s}^{H}(s)$ into portions parallel and perpendicular to $\boldsymbol{A}$, which itself is parallel to the displacement vector $\boldsymbol{Y}_{s}(t)-\boldsymbol{Y}_{s}(s)$. If we define the direction of the displacement vector by the unit vector $p(s)$, we can define the following:

$$
\begin{aligned}
& F_{s}^{H !}(s)=F_{s}^{H}(s) \cdot p(s) p(s), \\
& F_{s}^{H \perp}(s)=F_{s}^{H}(s) \cdot(I-p(s) p(s)),
\end{aligned}
$$

where

$$
p(s)=\frac{\boldsymbol{Y}_{s}(t)-\boldsymbol{Y}_{s}(s)}{\left|\boldsymbol{Y}_{s}(t)-\boldsymbol{Y}_{s}(s)\right|}
$$

Note that $\boldsymbol{F}_{s}^{H}(s)=\boldsymbol{F}_{s}^{H \|}(s)+\boldsymbol{F}_{s}^{H \perp}(s)$. By always letting $\boldsymbol{p}(s)$ point in the $z$-direction 
and $F_{s}^{H \perp}(s)$ in the $x$-direction in $\Omega$-space, the angular integration can be reduced to obtain

$$
\begin{aligned}
\boldsymbol{F}_{o s c}^{H}(t)= & \frac{3}{8}\left(\frac{\operatorname{ReS} l}{\pi}\right)^{\frac{1}{2}}\left\{\int _ { - \infty } ^ { t } \int _ { 0 } ^ { 1 } \left[2 ( 1 - x ^ { 2 } ) \left\{2 \boldsymbol{F}_{s}^{H \|}(s)(|\boldsymbol{A}| x)^{2} \mathrm{e}^{-(|\boldsymbol{A}| x)^{2}}\right.\right.\right. \\
& \left.+\left(\boldsymbol{F}_{s}^{H \|}(t)-\boldsymbol{F}_{s}^{H \|}(s) \mathrm{e}^{-(|\boldsymbol{A}| x)^{2}}\right)\right\} \\
& \left.+\left(1+x^{2}\right)\left\{2 \boldsymbol{F}_{s}^{H \perp}(s)(|\boldsymbol{A}| x)^{2} \mathrm{e}^{-(|A| x)^{2}}+\left(\boldsymbol{F}_{s}^{H \perp}(t)-\boldsymbol{F}_{s}^{H \perp}(s) \mathrm{e}^{-(|A| x)^{2}}\right)\right\}\right] \\
& \left.\times \frac{\mathrm{d} x \mathrm{~d} s}{(t-s)^{\frac{3}{2}}}\right\} \cdot \Phi .
\end{aligned}
$$

Here, $x$ represents the cosine of the angle between $p(s)$ and $n$. Finally, performing integration by parts in $x$, we find the following expression for $F_{O s c}^{H}(t)$ :

$$
\begin{aligned}
\boldsymbol{F}_{\text {osc }}^{H}(t)= & \frac{3}{8}\left(\frac{\operatorname{ReS} l}{\pi}\right)^{\frac{1}{2}}\left\{\int _ { - \infty } ^ { t } \left[\frac{2}{3} \boldsymbol{F}_{s}^{H \|}(t)-\left\{\frac{1}{|\boldsymbol{A}|^{2}}\left(\frac{\pi^{\frac{1}{2}}}{2|\boldsymbol{A}|} \operatorname{erf}(|\boldsymbol{A}|)-\exp \left(-|\boldsymbol{A}|^{2}\right)\right)\right\} \boldsymbol{F}_{s}^{H \|}(s)\right.\right. \\
& \left.+\frac{2}{3} \boldsymbol{F}_{s}^{H \perp}(t)-\left\{\exp \left(-|\boldsymbol{A}|^{2}\right)-\frac{1}{2|\boldsymbol{A}|^{2}}\left(\frac{\pi^{\frac{1}{2}}}{2|\boldsymbol{A}|} \operatorname{erf}(|\boldsymbol{A}|)-\exp \left(-|\boldsymbol{A}|^{2}\right)\right)\right\} \boldsymbol{F}_{s}^{H \perp}(s)\right] \\
& \left.\frac{2 \mathrm{~d} s}{(t-s)^{\frac{3}{2}}}\right\} \cdot \Phi .
\end{aligned}
$$

Now in order to investigate the temporal response of this correction, we evaluate (6.15) for various rectilinear slip velocities: $U_{s}(t)=U_{s}(t) p$. In all cases the timescale, $\tau_{c}$, is chosen as $4 v / U_{c}^{2}$ so that $R e S l=R e^{2} / 4$. This choice is simply for convenience since we are interested in long-time asymptotic behaviour. Also we let $F_{s}^{H \|}(t)=F_{s}^{H \|} U_{s}(t)$ and $F_{s}^{H \perp}(t)=F_{s}^{H \perp} U_{s}(t)$ where $F_{s}^{H \|}=-6 \pi(\Phi \cdot p) \cdot(p p)$ and $F_{s}^{H \perp}=-6 \pi(\Phi \cdot p) \cdot(I-p p)$.

First we consider the slip velocity given by a step change at $t=0, U_{s}(t)=H(t)$. After some manipulation and with the aid of Mathematica, we arrive at the following expression:

$$
\begin{aligned}
F_{O s c}^{H}(t)=\frac{3}{8} \operatorname{Re} \Phi \cdot[ & \boldsymbol{F}_{s}^{H \|}\left\{\left(1+\frac{1}{4 t^{2}}\right) \operatorname{erf}\left(t^{\frac{1}{2}}\right)+\frac{1}{(\pi t)^{\frac{1}{2}}}\left(1-\frac{1}{2 t}\right) \exp (-t)\right\} \\
& \left.+\boldsymbol{F}_{s}^{H \perp}\left\{\left(\frac{3}{2}-\frac{1}{8 t^{2}}\right) \operatorname{erf}\left(t^{\frac{1}{2}}\right)+\frac{3}{2(\pi t)^{\frac{1}{2}}}\left(1+\frac{1}{6 t}\right) \exp (-t)\right\}\right] .
\end{aligned}
$$

This result is consistent with that obtained by Sano, (1.5), for spherical particles. Recall that $\Phi=I$ for spheres. In addition, (6.16) demonstrates the existence of the $t^{-2}$ decay for arbitrarily shaped particles which confirms the scaling analysis in $\S 3$. Note that the above result does not include an 'added mass' contribution, which would appear simply as a delta function at $t=0$.

The expression given by (6.16) leads to a long-time asymptotic form for $\boldsymbol{F}_{o s c}^{H}(t)$ which is useful when a particle approaches its steady-state velocity monotonically from rest. Consider such a slip velocity, $U_{s}(t)$, which is zero for $t<0$ and goes to 1 as $t \rightarrow \infty$. Provided that for $O(1)$ values of $t$ on the timescale $4 v / U_{c}^{2}$ we have $\left|1-\left(Y_{s}(t)-Y_{s}(0)\right) / U_{s}(t) t\right| \ll 1$, the velocity profile will appear as a step change on this timescale. Under these conditions, the long-time asymptotic form can be predicted 
from (6.16) by replacing $U_{c}$ with $U_{c} U_{s}(t)$ in all terms implicitly scaled by $U_{c}$ :

$$
\begin{aligned}
\boldsymbol{F}_{o s c}^{H}(t)=\frac{3}{8} c \operatorname{Re} \Phi \cdot & {\left[\boldsymbol{F}_{s}^{H \|}\left\{\left(1+\frac{1}{4 c^{2} t^{2}}\right) \operatorname{erf}\left((c t)^{\frac{1}{2}}\right)+\frac{1}{(\pi c t)^{\frac{1}{2}}}\left(1-\frac{1}{2 c t}\right) \exp (-c t)\right\}\right.} \\
& \left.+\boldsymbol{F}_{s}^{H \perp}\left\{\left(\frac{3}{2}-\frac{1}{8 c^{2} t^{2}}\right) \operatorname{erf}\left((c t)^{\frac{1}{2}}\right)+\frac{3}{2(\pi c t)^{\frac{1}{2}}}\left(1+\frac{1}{6 c t}\right) \exp (-c t)\right\}\right],
\end{aligned}
$$

where $c(t)=U_{s}^{2}(t)$. Strictly speaking, this form corresponds to the result for a step change from zero to $U_{s}(t)$.

In order to test the validity of (6.17), for various $U_{s}(t)$ profiles we investigate the coefficient of the $\operatorname{Re} \Phi \cdot \boldsymbol{F}_{s}^{H \|}$-term extracted from the complete expression (6.15), defined here as $f(t)$ :

$f(t) \equiv \frac{3}{8} \frac{1}{\pi^{\frac{1}{2}}}\left\{\int_{-\infty}^{t}\left[\frac{2}{3} U_{s}(t)-\left\{\frac{1}{|\boldsymbol{A}|^{2}}\left(\frac{\pi^{\frac{1}{2}}}{2|\boldsymbol{A}|} \operatorname{erf}(|\boldsymbol{A}|)-\exp \left(-|\boldsymbol{A}|^{2}\right)\right)\right\} U_{s}(s)\right] \frac{\mathrm{d} s}{(t-s)^{\frac{3}{2}}}\right\}$.

Equation (6.17) indicates that by plotting $f(t) / c(t)$ versus $c(t) t$, the results should fall on the same curve. Since we wish to justify applying the asymptotic form to particles settling under gravity, we test it with trajectories that possess the long-time asymptotic form predicted by unsteady Stokes flow for a particle released from rest and settling under gravity:

$$
\begin{aligned}
U_{s}(t) & =0, & & t<0 \\
& =1-\frac{1}{(1+\pi t / R e S l)^{\frac{1}{2}}}, & & t>0 \\
& =1-\frac{1}{\left(1+4 \pi t / R e^{2}\right)^{\frac{1}{2}}}, & & t>0 .
\end{aligned}
$$

If we were to choose a trajectory with a $t^{-2}$ decay, it would not be surprising to see a force response as in (6.17) with a $t^{-2}$ decay. However, if we use a trajectory with a much slower decay, $t^{-\frac{1}{2}}$, as in (6.19), and the form (6.17) still holds, this will be a much more severe test since it is a greater deviation from a step-change velocity profile. The results (by numerical integration of (6.18)) for various values of $R e$ are given in figures 1 and 2 , which have unscaled and scaled axes, respectively. The curve for $R e=0$ corresponds to the step response given by (6.16). Figure 2 shows that the scaled variables collapse the results onto the $R e=0$ curve quite well. The fact that the curves are uniformly higher for increasing $R e$ reflects the increased deviation of the velocity profiles from that of a step change. Additional refinement can be achieved if, instead of $U_{s}^{2}(t)$, time was scaled with $\left(Y_{s}(t)-Y_{s}(0)\right)^{2} / t^{2}$, which is indicated by the presence of $\boldsymbol{A}$ in the exact result, (6.15).

Next, in order to test the generality of the $t^{-2}$ decay, we consider the temporal response of $f(t)$ to the following velocity profile:

$$
\begin{aligned}
U_{s}(t) & =b, & & t<0 \\
& =1, & & t>0,
\end{aligned}
$$




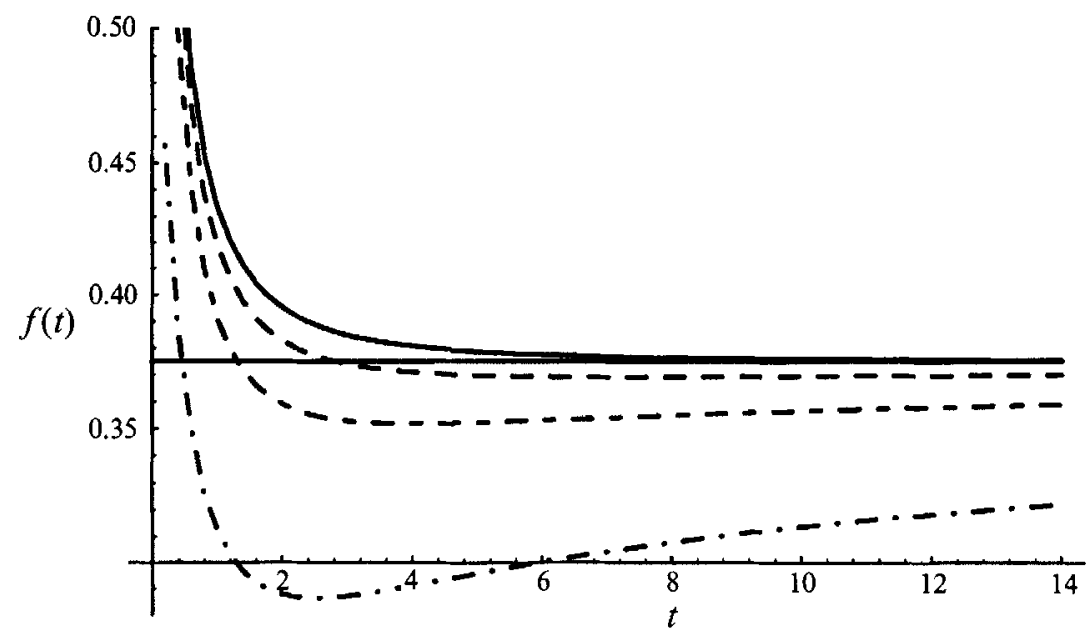

FIGURE 1. Temporal response of the unsteady Oseen correction to the hydrodynamic force, $f(t)$, due to a particle accelerating from rest as $O\left(t^{-\frac{1}{2}}\right)$. The particle trajectory is given by $(6.19)$ and $f(t)$ is defined by (6.18). Time is scaled by $4 v / U_{c}^{2}$. The curve for $R e=0$ is the step increase response from (6.16). The Reynolds number, which is a parameter in (6.19), is:,$- R e=0 ;--, R e=0.1$; ,$-- R e=0.3 ;-\cdot-, R e=1$.

where $0<b<1$. The results for various values of $b$ are shown in figure 3 . The exact solution for the case when $b=0$ is given by (6.16). The long-time asymptotic forms from (6.18) were found analytically to be

$$
\begin{array}{rlrl}
f(t) & =\frac{3}{8}\left[1+\left(\frac{1}{4}+O\left(t^{-\frac{1}{2}} \mathrm{e}^{-t}\right)\right) \frac{1}{t^{2}}\right], & b=0, & t \gg 1 \\
& =\frac{3}{8}\left[1+\left(\frac{1}{4(1-b)^{2}}+O\left(t^{-1}\right)\right) \frac{\mathrm{e}^{-4 b(1-b) t}}{t^{2}}\right], & 0<b<\frac{1}{2}, & t \gg \frac{(1-b)}{b(1-2 b)^{2}} \\
& =\frac{3}{8}\left[1+\left(\frac{1}{2}+O\left(t^{-\frac{1}{2}}\right)\right) \frac{\mathrm{e}^{-t}}{t^{2}}\right], & b=\frac{1}{2}, \quad t \gg 1 \\
& =\frac{3}{8}\left[1+\left(\frac{1-b}{2 b-1}+O\left(t^{-1}\right)\right) \frac{\mathrm{e}^{-t}}{\pi^{\frac{1}{2}} t^{\frac{5}{2}}}\right], & \frac{1}{2}<b<1, \quad t \gg \frac{1}{(1-2 b)^{2}} .
\end{array}
$$

We see that for a step change from a non-zero constant velocity, the decay to steady state is ultimately exponential. Note that for $b \ll 1, f(t)$ behaves as $(6.21 a)$ provided that $1 \ll t \ll 1 /(4 b)$. Also, when $(2 b-1) \ll 1, f(t)$ goes as $(6.21 c)$ if $1 \ll t \ll(2 b-1)^{-2}$.

Finally, we consider the velocity profile for a step change down:

$$
\begin{aligned}
U_{s}(t) & =1, & & t<0 \\
& =b, & & t>0 .
\end{aligned}
$$

The results for different values of $b$ are shown in figure 4 . The long-time asymptotic 


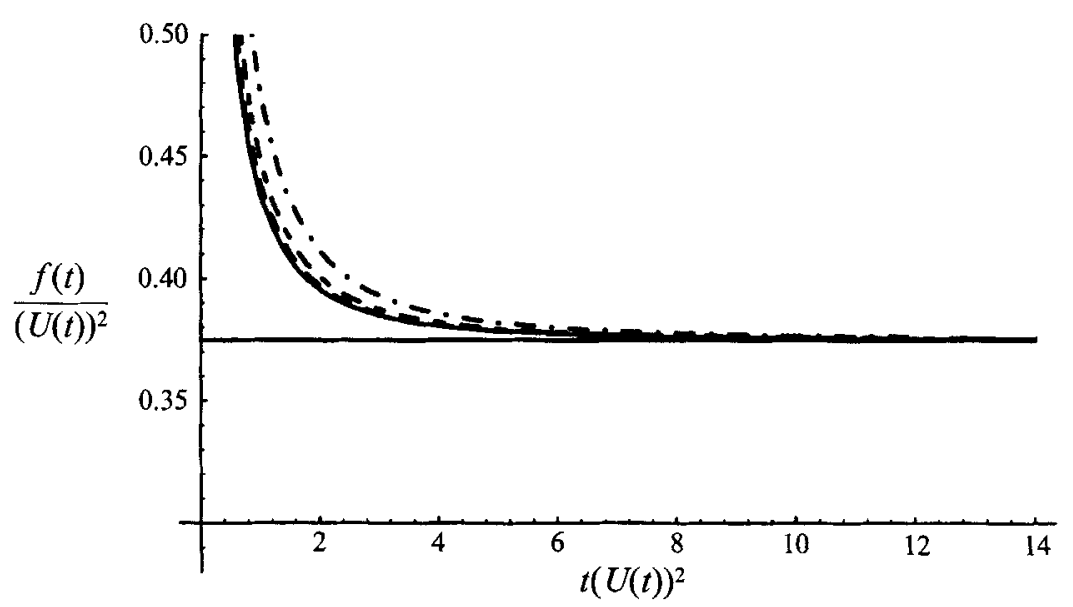

FIGURE 2. Temporal response of the scaled unsteady Oseen correction to the hydrodynamic force, $f(t) / U_{s}^{2}(t)$, due to a particle accelerating from rest as $O\left(t^{-\frac{1}{2}}\right)$. The particle trajectory is given by (6.19) and $f(t)$ is defined by (6.18). Time is scaled by $4 v / U_{c}^{2}$. The curve for $R e=0$ is the step increase response from (6.16). The Reynolds number, which is a parameter in (6.19), is: - $R e=0$; ,$-- \operatorname{Re}=0.1 ;---, \operatorname{Re}=0.3 ;-\cdot-, \operatorname{Re}=1$.

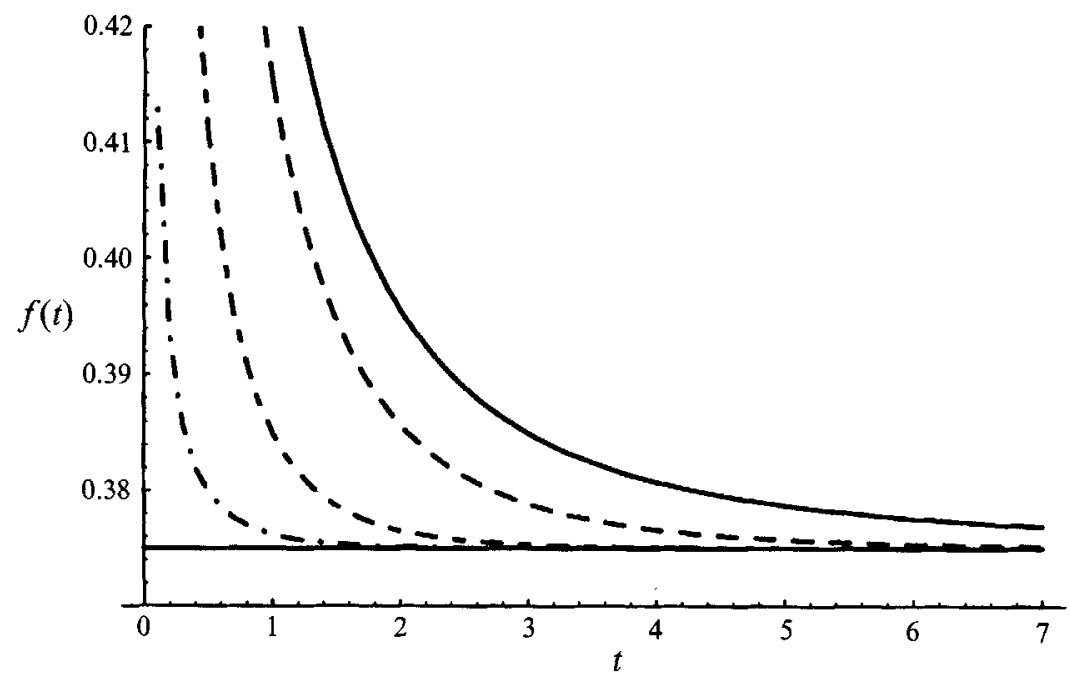

FIGURE 3. Temporal response of the unsteady Oseen correction to the hydrodynamic force, $f(t)$, due to a step change increase in the particle velocity. The particle trajectory is given by $(6.20)$ and $f(t)$ is defined by (6.18). Time is scaled by $4 v / U_{c}^{2}$. The ratio, $b$, of the particle velocity before the step change to that after is:,$- b=0 ;--, b=0.1 ;---, b=0.5 ;-\cdot-, b=0.9$.

forms from (6.18) were found analytically as

$$
\begin{aligned}
f(t) & =-\frac{3}{8}\left[\frac{1}{2 t}-\frac{1}{2 \pi^{\frac{1}{2}} t^{\frac{3}{2}}}+\frac{1}{8 t^{2}}-\frac{1}{16 \pi^{\frac{1}{2}} t^{\frac{5}{2}}}+O\left(t^{-3}\right)\right], \quad b=0, \quad t \gg 1 \\
& =\frac{3}{8} b^{2}\left[1-\left(\frac{1-b}{2-b}+O\left(\left(b^{2} t\right)^{-1}\right)\right) \frac{\mathrm{e}^{-b^{2} t}}{\pi^{\frac{1}{2}}\left(b^{2} t\right)^{\frac{5}{2}}}\right], \quad 0<b<1, \quad t \gg \frac{1}{b^{2}} .
\end{aligned}
$$

Equation (6.23a) also holds for $b \ll 1$ when $1 \ll t \ll b^{-2}$. 


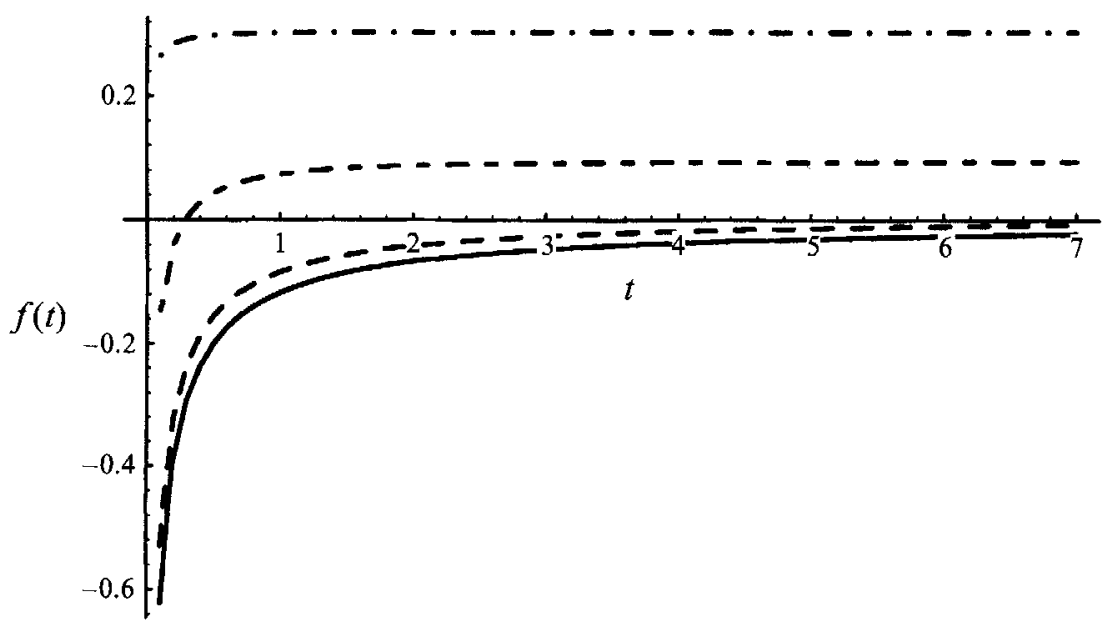

FIGURE 4. Temporal response of the unsteady Oseen correction to the hydrodynamic force, $f(t)$, due to a step change decrease in the particle velocity. The particle trajectory is given by $(6.22)$ and $f(t)$ is defined by (6.18). Time is scaled by $4 v / U_{c}^{2}$. The ratio, $b$, of the particle velocity after the step change to that before is: - $-b=0 ;--, b=0.1 ;---, b=0.5 ;-\cdot-, b=0.9$.

These differences in the temporal decay to steady state reflects the contrast between the creation or destruction of the wake structure, associated with the algebraic decay, and simply modifying the wake structure already established, which is associated with exponential decay. Step changes from or to zero velocity require the creation or complete destruction of the wake, and this evidently requires an algebraically long time. Step changes from and to finite velocities maintain the wake structure throughout the process, and the efficient convective transport of vorticity yields an exponential decay or response. These fundamentally different temporal responses - algebraic versus exponential - are quite intriguing and may have important implications for oscillatory flows or the response of particles to time-dependent fluctuating velocities caused, for example, by turbulent flows.

\section{Expression of the hydrodynamic force acting on a spherical particle}

The remaining contribution to the hydrodynamic force is given by

$$
\begin{aligned}
\boldsymbol{F}_{i n}^{H}(t)= & \boldsymbol{F}_{u s}^{H}(t)+\operatorname{ReSl} \widetilde{V}_{p} \dot{U}^{\infty}(t) \\
& -\operatorname{Re} \int_{V_{f}}\left(\operatorname{ReSl} \frac{\partial \boldsymbol{v}_{1}}{\partial t}+\boldsymbol{v}_{0} \cdot \nabla \boldsymbol{v}_{0}-U_{s}(t) \cdot \nabla \boldsymbol{v}_{0}\right) \cdot \boldsymbol{M} \mathrm{d} V .
\end{aligned}
$$

For a spherical particle, the evaluation of (7.1) is simple. The entire contribution from the regular perturbation is identically zero. This simplification follows as the $\boldsymbol{v}_{0}$-field is strictly symmetric, and the $\boldsymbol{v}_{1}$-field is strictly antisymmetric, as one can observe from their governing equations, (B 1) and (B 2). Hence, the entire integrand of the integral in (7.1) is antisymmetric. (One can note that the $\boldsymbol{M}$-field is symmetric from (3.3).) Then, since the volume of integration is spherically symmetric, angular integration will yield zero. For a sphere, the only contribution from (7.1) is the unsteady Stokes force, which is given by (1.2) with $U_{s}(t)$ replacing $U_{p}(t)$, and the buoyancy force 
$\operatorname{ReSl}\left(\widetilde{V}_{p} \dot{U}^{\infty}(t)\right.$ with $\widetilde{V}_{p}=\frac{4}{3} \pi$. Thus, we have

$$
F_{i n}^{H}(t)=-6 \pi\left\{U_{s}(t)+\left(\frac{R e S l}{\pi}\right)^{\frac{1}{2}} \int_{-\infty}^{t} \frac{\dot{U}_{s}(s)}{(t-s)^{\frac{1}{2}}} \mathrm{~d} s\right\}-\frac{2 \pi}{3} \operatorname{ReSl} \dot{U}_{s}(t)+\frac{4 \pi}{3} \operatorname{ReSl} \dot{U}^{\infty}(t) .
$$

Combining $\boldsymbol{F}_{\text {out }}^{H}(t)$ from (6.10) and (6.15) with (7.2), the expression for the total hydrodynamic force acting on a sphere is

$$
\begin{aligned}
\boldsymbol{F}^{H}(t)= & \frac{4 \pi}{3} \operatorname{ReSl} \dot{U}^{\infty}(t)-6 \pi U_{s}(t)-\frac{2 \pi}{3} \operatorname{ReSl} \dot{U}_{s}(t) \\
& +\frac{3}{8}\left(\frac{\operatorname{ReS} l}{\pi}\right)^{\frac{1}{2}}\left\{\int _ { - \infty } ^ { t } \left[\frac{2}{3} \boldsymbol{F}_{s}^{H \|}(t)-\left\{\frac{1}{|\boldsymbol{A}|^{2}}\left(\frac{\pi^{\frac{1}{2}}}{2|A|} \operatorname{erf}(|\boldsymbol{A}|)-\exp \left(-|\boldsymbol{A}|^{2}\right)\right)\right\} \boldsymbol{F}_{s}^{H \|}(s)\right.\right. \\
& \left.+\frac{2}{3} \boldsymbol{F}_{s}^{H \perp}(t)-\left\{\exp \left(-|\boldsymbol{A}|^{2}\right)-\frac{1}{2|\boldsymbol{A}|^{2}}\left(\frac{\pi^{\frac{1}{2}}}{2|\boldsymbol{A}|} \operatorname{erf}(|\boldsymbol{A}|)-\exp \left(-|A|^{2}\right)\right)\right\} \boldsymbol{F}_{s}^{H \perp}(s)\right] \\
& \left.\times \frac{2 \mathrm{~d} s}{(t-s)^{\frac{3}{2}}}\right\}+o(\operatorname{Re}),
\end{aligned}
$$

where we have used $\boldsymbol{F}_{s}^{H}(t)=-6 \pi \boldsymbol{U}_{s}(t)$. Thus, since the last term of (7.3) is $\boldsymbol{F}_{\text {osc }}^{H}(t)$, the asymptotic temporal behaviour described for the step changes in the previous section was actually for the entire hydrodynamic force.

Now we apply (7.3) to the problem of a sphere released from rest and settling under gravity. The equation of motion for a particle immersed in a fluid is given in dimensional form by

$$
m_{p} \dot{\boldsymbol{U}}_{p}(t)=\boldsymbol{F}^{\mathrm{ext}}(t)+\boldsymbol{F}^{H}(t)
$$

where $F^{e x t}(t)$ is the external force acting on the particle. For the problem of a particle settling under gravity, the external force is the buoyancy force

$$
\boldsymbol{F}^{\text {ext }}(t)=\left(m_{p}-m_{f}\right) g\left(-\boldsymbol{i}_{z}\right)
$$

where $m_{p}$ is the mass of the particle and $m_{f}$ is the mass of the fluid displaced by the particle. The hydrodynamic force acting on the sphere to $O(R e)$ is given by (7.3) with $U^{\infty}(t)=0, U_{s}(t)=U_{p}(t), Y_{s}(t)=Y_{p}(t)$, and $F_{s}^{H}(t)=-6 \pi U_{p}(t) . \dagger$ Thus, letting $\boldsymbol{U}_{p}(t)=U_{p}(t)\left(-\boldsymbol{i}_{z}\right), \boldsymbol{Y}_{p}(t)=Y_{p}(t)\left(-\boldsymbol{i}_{z}\right)$, and $\boldsymbol{F}^{H}(t)=F^{H}(t)\left(-\boldsymbol{i}_{z}\right)$, we have for the sphere released from rest at $t=0$

$$
\begin{aligned}
\frac{F^{H}(t)}{\mu a U_{c}}= & -6 \pi U_{p}(t)-\frac{2}{3} \pi \operatorname{ReSl} \dot{U}_{p}(t) \\
& -\frac{9}{4}(\pi R e S l)^{\frac{1}{2}}\left\{\int_{0}^{t}\left[\frac{2}{3} U_{p}(t)-\left\{\frac{1}{A^{2}}\left(\frac{\pi^{\frac{1}{2}}}{2 A} \operatorname{erf}(A)-\exp \left(-A^{2}\right)\right)\right\} U_{p}(s)\right]\right. \\
& \left.\times \frac{2 \mathrm{~d} s}{(t-s)^{\frac{3}{2}}}+\frac{8 U_{p}(t)}{3 t^{\frac{1}{2}}}\right\},
\end{aligned}
$$

$\dagger$ In fact we could equally well replace $\boldsymbol{F}_{s}{ }^{H}(t)$ with the full force monopole, $\boldsymbol{F}_{1}(t)$, given by (B 7). In this case, $\boldsymbol{F}_{1}(t)=-\boldsymbol{F}^{\text {ext }}+\left(m_{p} / m_{j}-1\right) \widetilde{V}_{p} \operatorname{ReSl} \dot{U}_{p}(t) \approx-\boldsymbol{F}^{\text {ext }}$ for $\tau_{c}=v / \boldsymbol{U}_{c}^{2}$. However, we then would need to include the Basset term and the quantity $(R e S l / \pi t)^{\frac{1}{2}} F^{\text {ext }}$ as well in (7.3). Regardless, the difference in choice will alter the result by an amount smaller than $O(R e)$. 


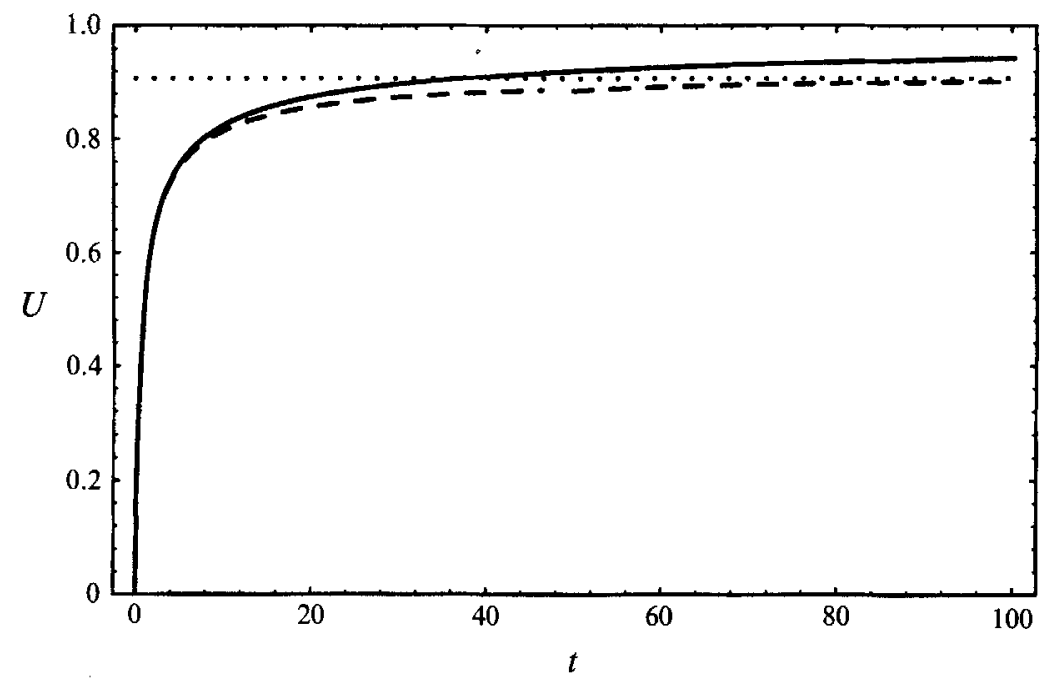

FIGURE 5. Trajectory of a sphere released from rest and settling under gravity. The ratio of particle density to fluid density, $\rho_{p} / \rho_{f}$, is 1.1 . Time is scaled by $a^{2} / v$ and the velocity by the Stokes terminal velocity. The right side of the curve for $R e=0.3$ is from the long-time asymptotic result given by (7.10). The Reynolds number is: - $R e=0 ;--, R e=0.3 ; \ldots, R e=0.3$ (steady state solution).

where

$$
A^{2}=\frac{R e^{2}}{4} \frac{t-s}{\operatorname{ReSl} l}\left(\frac{Y_{p}(t)-Y_{p}(s)}{t-s}\right)^{2}
$$

Combining (7.5) and (7.6) in (7.4) and rearranging, we obtain for the equation of motion of the sphere

$$
\begin{aligned}
1= & U_{p}(t)+\frac{1}{9}\left(1+2 \frac{\rho_{p}}{\rho}\right) \dot{U}_{p}(t) \\
& +\frac{3}{8 \pi^{\frac{1}{2}}}\left\{\int_{0}^{t}\left[\frac{2}{3} U_{p}(t)-\left\{\frac{1}{A^{2}}\left(\frac{\pi^{\frac{1}{2}}}{2 A} \operatorname{erf}(A)-\exp \left(-A^{2}\right)\right)\right\} U_{p}(s)\right]\right. \\
& \left.\times \frac{2 \mathrm{~d} s}{(t-s)^{\frac{3}{2}}}+\frac{8 U_{p}(t)}{3 t^{\frac{1}{2}}}\right\},
\end{aligned}
$$

where $\rho_{p}$ is the density of the particle. Here, we have taken the diffusive timescale, $a^{2} / v$, so that $\operatorname{Re} S l=1$, and the Stokes terminal velocity as the velocity scale:

$$
U_{c}=U_{\text {Stokes }}=\frac{\left(m_{p}-m_{f}\right) g}{6 \pi \mu a}
$$

We note that $R e=a U_{c} / v=2\left(\rho_{p}-\rho\right) \rho g a^{3} / 9 \mu^{2}$. Also, with $U_{p}(0)=0$, we have

$$
\dot{U}_{p}(0)=\frac{9}{1+2 \rho_{p} / \rho} .
$$

With a simple time-stepping, finite-difference routine $U_{p}(t)$ was solved numerically to produce figure 5, with $R e=0.3$ and $\rho_{p} / \rho=1.1$. This value of $R e$ was chosen based on experimental results which showed the steady Oseen formula for the drag to be a good approximation for Reynolds numbers below 0.4 (Maxworthy 1965). The curve for pure unsteady Stokes flow (i.e. for $R e=0$ ) is included for comparison. By 
definition, the terminal velocity associated with this curve is 1.0 . Numerical difficulties occurred for the $R e=0.3$ curve after $t \sim 50$ which are believed to be due to the nonlinearity of the governing equation (7.7). The remainder of the curve was obtained using the long-time asymptotic formula (6.17) for the last term of (7.7), the unsteady Oseen correction. Using (6.17) for $t \gg R e^{-2}$ (note that here $t$ must be replaced by $R e^{2} t / 4$ in (6.17)), we have the following solution for $U_{p}(t)$ :

$$
U_{p}(t) \sim U_{p_{0}}-\frac{\frac{3}{2} R e}{\left(\operatorname{Re}^{2} t\right)^{2}}\left[\frac{1}{U_{p_{0}}\left(2-U_{p_{0}}\right)}\right]+o\left(\left(R e^{2} t\right)^{-2}\right),
$$

where

$$
U_{p_{0}}=\frac{4}{3 R e}\left(\left(1+\frac{3}{2} R e\right)^{\frac{1}{2}}-1\right) .
$$

We find that for $R e=0.3$ the decay to steady state (defined as $99 \%$ of the terminal velocity) is about forty times faster with the unsteady Oseen correction than with just the unsteady Stokes force. One must be cautious with such conclusions, however, since this sort of temporal decay is bounded by the existence of higher-order terms which may have slower than $O\left(t^{-2}\right)$ decay. We note that the next correction, which is $O\left(R e^{2} \log R e\right)$, was indicated by Sano (1981) to have no temporal nature. This is believed to be related to the fact that the $O\left(R e^{2} \log R e\right)$ term arises from volume integration in the reciprocal theorem of the inner fields over the inner region where the fluid motion is nearly steady. $\dagger$

\section{Expression of the hydrodynamic force acting on an arbitrarily shaped particle: long-time limit}

As can be seen from (7.1), to evaluate the hydrodynamic force to $O(R e)$ in general would require both the unsteady Stokes velocity field, $\boldsymbol{v}_{0}$, and its regular perturbation, $v_{1}$. Computing such fields for non-spherical particles is a difficult task. Few solutions exist for the unsteady Stokes field created by the motion of a non-spherical particle. Most notable is the rather complex formulation presented by Lawrence \& Weinbaum (1986) for the axisymmetric motion of a spheroid. We are unaware, however, of any solutions for the regular perturbation field.

For a non-spherical particle, however, one can use the general approach of the reciprocal theorem and the idea of a uniformly valid velocity field to find the form of the unsteady Stokes corrections to the pseudo-steady Stokes drag in the limit of $R e S l \ll 1$. This is the limit when the time scale of variation is much longer than the diffusive scale $a^{2} / v$. The Basset history force in the long-time limit, which, in general, may be different in the short-time limit, is identified as the term that is $O\left((\operatorname{ReSl})^{\frac{1}{2}}\right)$. The acceleration reaction (for lack of a better name) will represent the $O(R e S l)$ term since, as we shall see below, it is proportional to the particle acceleration. These terms

$\dagger$ One can compute the $O\left(R e^{2} \log R e\right)$ term from:

$$
-\boldsymbol{R} e^{2} \int_{r=O(1)}^{r=O\left(R e^{-1}\right)}\left[-U_{s}(t) \cdot \nabla u_{1}^{-1}+u_{0}^{-1} \cdot \nabla u_{1}^{0}+u_{1}^{0} \cdot \nabla u_{0}^{-1}\right] \cdot M_{p} \mathrm{~d} \boldsymbol{V}
$$

where $\boldsymbol{u}_{1}$ is the solution to the regular perturbation to steady Stokes flow and the superscripts indicate that the velocity fields are homogeneous in that power of $|\boldsymbol{r}|$, i.e. $\boldsymbol{u}_{1}^{-1}$ represents those terms of $\boldsymbol{u}_{1}$ that are $O\left(r^{-1}\right)$. By analogy, we anticipate that this term will be $O\left(\operatorname{Re} e^{2} \log \left((\operatorname{ReSl})^{-\frac{1}{2}}\right)\right)$ for timescales such that $a^{2} / v \ll \tau_{c} \ll v / U_{c}^{2}$, since the velocity fields in the integral are only valid out to the distance to which vorticity has diffused, $O\left((\operatorname{ReS} l)^{-\frac{1}{2}}\right)$. 
are contained in $\boldsymbol{F}_{u s}^{H}$, so for now we only consider this term and not the integral in (7.1).

We begin with the reciprocal theorem expression for the unsteady Stokes force from (5.2):

$$
\boldsymbol{F}_{u s}^{H}(t)=\boldsymbol{F}_{s}^{H}(t)-\operatorname{Re} S l \int_{V_{f}} \frac{\partial \boldsymbol{v}_{0}}{\partial t} \cdot \boldsymbol{M} \mathrm{d} V,
$$

where $v_{0}$ is the unsteady Stokes velocity field (i.e. the solution of (B 1)). When $\operatorname{ReSl} \ll 1$, near to the particle the velocity field will be dominated by steady Stokes flow, while far from the particle at distances of $O\left(a(\operatorname{ReSl})^{-\frac{1}{2}}\right)$, the flow to leading order is given by the point-forced unsteady Stokes solution. Thus, we approximate $v_{0}$ with a uniformly valid velocity field as

$$
\boldsymbol{v}_{0} \sim \boldsymbol{v}_{0}^{u v}=\left(\boldsymbol{u}_{0}-\boldsymbol{u}_{0}^{p}\right)+\boldsymbol{v}^{p},
$$

where $\boldsymbol{u}_{0}$ is the steady Stokes velocity field, and $\boldsymbol{u}_{0}^{p}$ is the solution to the point-forced Stokes field given by (B 13). The solution to the full point-forced unsteady Stokes velocity field, $\boldsymbol{v}^{p}$, is given by the solution to

$$
-\nabla \Pi^{p}+\nabla^{2} v^{p}=\operatorname{ReSl} \frac{\partial v^{p}}{\partial t}+F_{1}^{u s}(t) \delta(r), \quad \nabla \cdot v^{p}=0,
$$

which is identified as the outer velocity field. Here we retain the entire force monopole to ensure the desired accuracy in the hydrodynamic force; that is,

$$
F_{1}^{u s}(t)=F_{u s}^{H}(t)-\operatorname{ReSl} \widetilde{V}_{p} \dot{U}_{s}(t)
$$

In the presence of an $O(1)$ dipole contribution to the velocity field, which would arise from an external torque or straining motion, this uniformly valid field is not accurate to $O(\operatorname{ReSl})$, but only to $O\left((\operatorname{ReSl})^{\frac{1}{2}}\right)$. We assume for now that there is no dipole contribution, but will return to this issue later.

If we define $\boldsymbol{M}_{\boldsymbol{s}}$ as

and note that

$$
\boldsymbol{M}_{s}=\boldsymbol{M}-\boldsymbol{M}_{p}
$$

$$
\boldsymbol{u}_{0}-\boldsymbol{u}_{0}^{p}=\left(\boldsymbol{M}-\boldsymbol{M}_{p}\right) \cdot \boldsymbol{U}_{s}(t)
$$

then (8.2) becomes

$$
\boldsymbol{v}_{0}^{u v}=\boldsymbol{M}_{\mathrm{s}} \cdot \boldsymbol{U}_{\mathrm{s}}(t)+\boldsymbol{v}^{p} .
$$

Using this approximation for the unsteady Stokes velocity field we obtain the following expression for the unsteady Stokes drag to $O(R e S l)$ :

$$
\boldsymbol{F}_{u s}^{H}(t)=\boldsymbol{F}_{s}^{H}(t)-\operatorname{ReSl} \dot{\boldsymbol{U}}_{s}(t) \cdot \int_{V_{f}} \boldsymbol{M}_{s}^{T} \cdot \boldsymbol{M} d V-\operatorname{ReSl} \int_{V_{f}} \frac{\partial \boldsymbol{v}^{p}}{\partial t} \cdot \boldsymbol{M} d V+o(\operatorname{Re} S l),
$$

where $\boldsymbol{M}_{s}^{T}$ is the transpose of $\boldsymbol{M}_{s}$.

One can see that if there were a dipole contribution to $\boldsymbol{M}_{\boldsymbol{s}}$, the first integral of (8.8) would be conditionally convergent, as the dipole is antisymmetric and $O\left(r^{-2}\right)$, while the monopole contribution from $\boldsymbol{M}$ is $O\left(r^{-1}\right)$ and symmetric. That is, if we perform the radial integration first we obtain a log singularity at infinity; while angular integration, done first over any spherical surface, removes the log singularity. Note that without the dipole $\boldsymbol{M}_{s}$ is $\boldsymbol{O}\left(r^{-3}\right)$.

This apparent problem is simply a shortcoming of approximating the actual velocity by a field to too low an accuracy in $R e S l$, only to $O\left((\operatorname{ReSl})^{\frac{1}{2}}\right)$, in the outer region. To be mathematically rigorous, one could include the dipole field in the outer velocity 
field in the same manner as was done for the monopole. This creates unnecessary labour, however. Instead, if there is a dipole contribution, we may force a convergent order of integration by writing $(8.8)$ as

$$
\boldsymbol{F}_{u s}^{H}(t)=\boldsymbol{F}_{s}^{H}(t)-\operatorname{ReS} l \dot{\boldsymbol{U}}_{s}(t) \cdot \lim _{R \rightarrow \infty} \int_{V_{f}(R)} \boldsymbol{M}_{s}^{T} \cdot \boldsymbol{M} \mathrm{d} V-\operatorname{ReSl} \int_{V_{f}} \frac{\partial \boldsymbol{v}^{p}}{\partial t} \cdot \boldsymbol{M} \mathrm{d} V+o(\operatorname{ReSl}),
$$

where now $V_{f}(R)$ is the volume of fluid surrounding the particle and bounded by a spherical surface of radius $R$. This is justified by the fact that the actual dipole contribution to the unsteady Stokes field is antisymmetric but only $O\left(r^{-4}\right)$ for large $r$. As an added note, a dipole contribution will only exist if the particle can exert a torque (or stresslet) on the fluid by its translational motion. This would be the case, for example, for a screw-shaped particle if there was an external torque to prevent it from rotating.

Now we shall consider how to simplify the calculation of the second integral of (8.9), the contribution of $\boldsymbol{v}^{p}$ to $\boldsymbol{F}_{u s}^{H}$. First we decompose it into the following two parts:

$$
\operatorname{ReSl} \int_{V_{f}} \frac{\partial \boldsymbol{v}^{p}}{\partial t} \cdot \boldsymbol{M} \mathrm{d} V=\operatorname{ReS} l \int_{V_{f}} \frac{\partial \boldsymbol{v}^{p}}{\partial t} \cdot \boldsymbol{M}_{p} \mathrm{~d} V+\operatorname{ReS} l \int_{V_{f}} \frac{\partial \boldsymbol{v}^{p}}{\partial t} \cdot \boldsymbol{M}_{s} \mathrm{~d} V
$$

In the last integral of $(8.10)$, we may replace $\boldsymbol{v}^{p}$ with $\boldsymbol{u}_{0}^{p}$ because in the outer region, where this is an invalid approximation, the error that it makes in the hydrodynamic force is $o(\operatorname{ReSl})$. Here again the dipole contribution may be dealt with as above to obtain

$$
\operatorname{ReSl} \int_{V_{f}} \frac{\partial \boldsymbol{v}^{p}}{\partial t} \cdot \boldsymbol{M}_{s} \mathrm{~d} V=\operatorname{ReSl} \dot{\boldsymbol{U}}_{s}(t) \cdot \lim _{R \rightarrow \infty} \int_{V_{f}(R)} \boldsymbol{M}_{p}^{T} \cdot \boldsymbol{M}_{s} \mathrm{~d} V+o(\operatorname{Re} S l)
$$

The first integral in (8.10) may be rewritten as an integral over the entire region of space minus that over the volume of the particle and approximated as

$$
\begin{array}{r}
\operatorname{ReSl} \int_{V_{f}} \frac{\partial \boldsymbol{v}^{p}}{\partial t} \cdot \boldsymbol{M}_{p} \mathrm{~d} V=\operatorname{ReSl} \int_{V_{\infty}} \frac{\widehat{\partial \boldsymbol{v}^{p}}}{\partial t}(\boldsymbol{k}) \cdot \widehat{\boldsymbol{M}_{p}}(-\boldsymbol{k}) \mathrm{d} \boldsymbol{k}-\operatorname{ReSl} \dot{\boldsymbol{U}}_{s}(t) \cdot \int_{V_{p}} \boldsymbol{M}_{p}^{T} \cdot \boldsymbol{M}_{p} \mathrm{~d} V \\
+o(\operatorname{Re} S l)
\end{array}
$$

In the integral over the volume of the particle we have replaced $\boldsymbol{v}^{p}$ with $\boldsymbol{u}_{0}^{p}$, since this makes an error that is $o(R e S l)$. The integral over all space was rewritten in Fourier space with the use of the convolution theorem. Combining (8.11) and (8.12) in (8.10), this contribution to the unsteady Stokes force becomes

$$
\begin{aligned}
-\operatorname{ReSl} \int_{V_{f}} \frac{\partial \boldsymbol{v}^{p}}{\partial t} \cdot \boldsymbol{M} \mathrm{d} \boldsymbol{V}= & -\operatorname{ReSl} \int_{V_{\infty}} \frac{\widehat{\partial \boldsymbol{v}^{p}}}{\partial t}(\boldsymbol{k}) \cdot \widehat{\boldsymbol{M}_{p}}(-\boldsymbol{k}) \mathrm{d} \boldsymbol{k}+\operatorname{ReSl} \dot{\boldsymbol{U}}_{s}(t) \cdot \int_{V_{p}} \boldsymbol{M}_{p}^{T} \cdot \boldsymbol{M}_{p} \mathrm{~d} V \\
& -\operatorname{ReSl} \dot{\boldsymbol{U}}_{s}(t) \cdot \lim _{R \rightarrow \infty} \int_{V_{f}(R)} \boldsymbol{M}_{p}^{T} \cdot \boldsymbol{M}_{s} \mathrm{~d} V+o(\operatorname{ReSl})
\end{aligned}
$$

The integral in (8.13) involving the Fourier transforms can be evaluated in the same manner as was done in $\S 6$. Following the procedure in Appendix C, we obtain

$$
\operatorname{ReSl} \frac{\widehat{\partial \boldsymbol{v}^{p}}}{\partial t}=\int_{-\infty}^{t} \dot{\boldsymbol{F}}_{1}^{u s}(s) \cdot(\boldsymbol{n} n-I) \mathrm{e}^{-4 \pi^{2} k^{2}(t-s) / \operatorname{Resl} \mathrm{d} s}
$$


Combining (6.4) for $\widehat{\boldsymbol{M}}_{p}$ and (8.14) and performing the integration over $\boldsymbol{k}$-space gives

$$
\operatorname{ReSl} \int_{V_{\infty}} \frac{\widehat{\partial \boldsymbol{v}^{p}}}{\partial t}(\boldsymbol{k}) \cdot \widehat{\boldsymbol{M}_{p}}(-\boldsymbol{k}) \mathrm{d} \boldsymbol{k}=-\left(\frac{\operatorname{ReSl}}{\pi}\right)^{\frac{1}{2}} \int_{-\infty}^{t} \frac{\dot{\boldsymbol{F}}_{1}^{u s}(\mathrm{~s})}{(t-s)^{\frac{1}{2}}} \mathrm{~d} \cdot \boldsymbol{\Phi} .
$$

Now we must consider carefully what form $\boldsymbol{F}_{1}^{u s}(t)$ must take in order to obtain the hydrodynamic force to $O(R e S l)$. Note that $F_{1}^{u s}(t)$ represents the exact hydrodynamic force acting on the particle due to the unsteady Stokes field plus a term that is $O(R e S l)$. To obtain the contribution to ihe hydrodynamic force from (8.15) to $O(R e S l), F_{1}^{u s}(t)$ must be replaced with the particle drag to $O\left((\operatorname{ReSl})^{\frac{1}{2}}\right)$. The $O(1)$ contribution is the pseudo-steady Stokes drag, $\boldsymbol{F}_{s}^{H}(t)$. The $O\left((\operatorname{Re} S l)^{\frac{1}{2}}\right)$ contribution to $\boldsymbol{F}_{1}^{u s}(t)$ can be found by evaluating (8.15) with $\boldsymbol{F}_{1}^{u s}(t)$ replaced by $\boldsymbol{F}_{s}^{H}(t)$. Thus the proper form of $\boldsymbol{F}_{1}^{u s}(t)$ to be used in (8.15) is

$$
\boldsymbol{F}_{1}^{u s}(t)=\boldsymbol{F}_{s}^{H}(t)+\left(\frac{\operatorname{ReS} l}{\pi}\right)^{\frac{1}{2}} \int_{-\infty}^{t} \frac{\dot{\boldsymbol{F}}_{s}^{H}(s)}{(t-s)^{\frac{1}{2}}} \mathrm{~d} s \cdot \boldsymbol{\Phi}+o\left((\operatorname{ReS} l)^{\frac{1}{2}}\right) .
$$

Evaluating (8.15), using the above expression for $\boldsymbol{F}_{1}^{u s}(t)$ we obtain

$$
\begin{aligned}
-\operatorname{ReSl} \int_{V_{\infty}} \frac{\widehat{\partial \boldsymbol{v}^{p}}}{\partial t} \cdot \widehat{\boldsymbol{M}_{p}} d \boldsymbol{k}= & \left(\frac{\operatorname{ReS} l}{\pi}\right)^{\frac{1}{2}} \int_{-\infty}^{t} \frac{\dot{\boldsymbol{F}}_{s}^{H}(s)}{(t-s)^{\frac{1}{2}}} \mathrm{~d} s \cdot \Phi \\
& +\operatorname{ReS} S l \dot{\boldsymbol{F}}_{s}^{H}(t) \cdot \boldsymbol{\Phi} \cdot \boldsymbol{\Phi}+o(\operatorname{ReS} l) .
\end{aligned}
$$

Finally, combining the above result with (8.13) in the expression of the total unsteady Stokes force (8.9), we obtain, correct to $O(\operatorname{ReSl})$,

$$
\begin{aligned}
\boldsymbol{F}_{u s}^{H}(t)= & -6 \pi \Phi \cdot\left\{\boldsymbol{U}_{s}(t)+\left(\frac{\operatorname{ReSl} l}{\pi}\right)^{\frac{1}{2}} \Phi \cdot \int_{-\infty}^{t} \frac{\dot{U}_{s}(s)}{(t-s)^{\frac{1}{2}}} \mathrm{~d} s+\operatorname{ReSl} \boldsymbol{\Phi} \cdot \boldsymbol{\Phi} \cdot \dot{U}_{s}(t)\right\} \\
& -\operatorname{ReSl}\left\{\lim _{R \rightarrow \infty}\left(\int_{V_{f}(R)} \boldsymbol{M}^{T} \cdot \boldsymbol{M} \mathrm{d} V-\frac{9 \pi}{2} \Phi \cdot \Phi R\right)\right\} \cdot \dot{U}_{s}(t) \\
& +o(\operatorname{ReSl}),
\end{aligned}
$$

where we have used the fact that $\int_{V_{f}(R)+V_{p}} \boldsymbol{M}_{p}^{T} \cdot \boldsymbol{M}_{p} \mathrm{~d} V=\frac{9 \pi}{2} \boldsymbol{\Phi} \cdot \boldsymbol{\Phi} R$. The first term is the pseudo-steady Stokes drag. The second is identified as the Basset history force in the long-time limit. This form of the history force was first noted by Williams (1966). The remaining terms combine to contribute to what we have referred to as the acceleration reaction, being proportional to the particle acceleration relative to the imposed flow. It is the counterpart to the added mass in the short-time limit associated with potential flow. The entire result agrees in form with the expression obtained by Pozrikidis (1989) for the low-frequency oscillation of a particle. However, Pozrikidis' expression requires the solution of an integral equation for the given particle. It is also interesting to point out that the above resultant 'acceleration reaction' resistance tensor is symmetric, which agrees with the finding of Gavze (1990).

To find the acceleration reaction correction in this limit of small ReSl, we only require the steady Stokes velocity field created by the translating particle at time $t$ and the corresponding Stokes drag. Indeed, if we use the steady Stokes velocity field for a translating sphere we obtain $-\frac{2}{3} \pi \operatorname{ReSl} \dot{U}_{p}(t)$, the added mass of a sphere, which agrees with the fact that the acceleration reaction and the added mass are the same for the special case of a spherical particle. If we use the Stokes velocity field and drag for an oblate spheroid translating along its axis of symmetry, given in the text 
by Happel \& Brenner (1986), we obtain the following expression for the acceleration reaction in dimensional variables when $\operatorname{ReSl} \ll 1$ :

$$
\begin{aligned}
\boldsymbol{F}_{\text {acc }}^{H}(t)= & -\frac{2}{3} \pi \rho b^{3} \dot{U}_{s}(t)\left\{9\left(\frac{\frac{4}{3} \lambda^{-1}}{\lambda-\left(\lambda^{2}-1\right) \cot ^{-1} \lambda}\right)^{3}\right. \\
& +\frac{2 \lambda^{-3}}{\left(\lambda-\left(\lambda^{2}-1\right) \cot ^{-1} \lambda\right)^{2}}\left[2 \lambda+4 \lambda^{3}+\left(2+5 \lambda^{2}-3 \lambda^{6}\right) \cot ^{-1} \lambda\right. \\
& \left.\left.-\left(1-2 \lambda^{2}+\lambda^{4}\right)\left(\int_{\lambda}^{\infty}\left[\left(\cot ^{-1} x\right)^{2}+3\left(x \cot ^{-1} x\right)^{2}-3\right] \mathrm{d} x-3 \lambda\right)\right]\right\},
\end{aligned}
$$

where

$$
\lambda=b /\left(a^{2}-b^{2}\right)^{\frac{1}{2}} .
$$

Here $a$ and $b$ the are major and minor semi-axes of the spheroid, respectively. For a slightly oblate spheroid we define its non-sphericity, $\epsilon$, by

$$
a=b(1+\epsilon), \quad \epsilon \ll 1 .
$$

Then the acceleration reaction becomes

$$
F_{a c c}^{H}(t)=-\frac{2}{3} \pi \rho b^{3}\left\{1+\frac{16}{5} \epsilon+\frac{628}{175} \epsilon^{2}+o\left(\epsilon^{2}\right)\right\} \dot{U}_{s}(t),
$$

which agrees with the result of Lawrence \& Weinbaum (1986) in the low-frequency limit of an oscillating slightly oblate spheroid for the part of the force proportional to the first power of the frequency. We note that the added mass found by Lawrence \& Weinbaum (1986) in the high-frequency limit differs from (8.21) at $O\left(\epsilon^{2}\right)$, which again demonstrates the uniqueness of the result (1.2) for a perfect solid sphere.

Finally, for $R e S l \ll 1$, the contribution to the hydrodynamic force from the regular perturbation to unsteady Stokes flow (the integral portion of (7.1)) may be simplified. Clearly, the part from the term $\operatorname{Re}(\operatorname{ReSl}) \partial \boldsymbol{v}_{1} / \partial t$ is only a small correction to the already small $O(R e)$ correction to the drag evaluated in $\$ 6$ and can therefore be neglected. In addition, in the terms involving $\operatorname{Rev}_{0} \cdot \nabla v_{0}$ and $\operatorname{Re} U_{s}(t) \cdot \nabla v_{0}, v_{0}$ could be replaced with the approximate field given by (8.2). However, even this level of accuracy is unnecessary. If we simply use the steady Stokes field, $\boldsymbol{u}_{0}$, we can obtain the correction to $O(R e)$, but to only $O(1)$ in $R e S l$. In doing so, however, one must recognize that the integrand, $\operatorname{Re} U_{s}(t) \cdot \nabla \boldsymbol{u}_{0} \cdot \boldsymbol{M}$, is conditionally convergent, since it is $O\left(r^{-3}\right)$ and antisymmetric at large distances from the particle. Thus, we simply approximate this entire contribution by writing

$$
\begin{aligned}
\operatorname{Re} \int_{V_{f}}(\operatorname{ReSl} & \left.\frac{\partial \boldsymbol{v}_{1}}{\partial t}+v_{0} \cdot \nabla v_{0}-U_{s}(t) \cdot \nabla \boldsymbol{v}_{0}\right) \cdot \boldsymbol{M} \mathrm{d} V \\
& =\operatorname{Re} \lim _{R \rightarrow \infty} \int_{V_{f}(R)}\left(u_{0} \cdot \nabla u_{0}-U_{s}(t) \cdot \nabla u_{0}\right) \cdot \boldsymbol{M} \mathrm{d} V+o(\operatorname{Re}) .
\end{aligned}
$$

The integral on the right-hand side of (8.22) may be shown to only contribute a force orthogonal to the direction of motion of the particle - a lift force. The portion parallel to the direction of motion is given by the volume integration of

$$
\begin{aligned}
\left(\boldsymbol{u}_{0} \cdot \nabla \boldsymbol{u}_{0}-\boldsymbol{U}_{s}(t) \cdot \nabla \boldsymbol{u}_{0}\right) \cdot \boldsymbol{M} \cdot \boldsymbol{U}_{s}(t) & =\left[\nabla \cdot\left(\boldsymbol{u}_{0} \boldsymbol{u}_{0}-\boldsymbol{U}_{s}(t) \boldsymbol{u}_{0}\right)\right] \cdot \boldsymbol{u}_{0} \\
& =\frac{1}{2} \boldsymbol{\nabla} \cdot\left(\boldsymbol{u}_{0} \boldsymbol{u}_{0} \cdot \boldsymbol{u}_{0}-U_{s}(t) \boldsymbol{u}_{0} \cdot \boldsymbol{u}_{0}\right),
\end{aligned}
$$


where we have used the continuity equation and that $\boldsymbol{M} \cdot \boldsymbol{U}_{s}(t)=\boldsymbol{u}_{0}$. Application of the divergence theorem in the volume integral of this quantity and using the boundary condition on the surface of the particle yields surface integrals which are identically zero. Thus, the contribution given by (8.22) can only yield a transverse or side force. This result agrees with the conclusion reached by Brenner \& Cox (1963). This form of the term represented by (8.22) was first noted in a paper by Cox (1965).

If we combine the results of $\S \S 6$ and 8 , we obtain the expression of the hydrodynamic force acting on an arbitrarily shaped rigid particle in the limit when the time scale associated with the particle's slip velocity is much greater than the diffusive scale. Combining (6.10) and (6.15) with (7.1), (8.18), and (8.22) the hydrodynamic force is

$$
\begin{aligned}
\boldsymbol{F}^{H}(t)= & \operatorname{ReSl} \tilde{V}_{p} \dot{U}^{\infty}(t)+\boldsymbol{F}_{s}^{H}(t) \\
& -\operatorname{ReSl}\left\{6 \pi \Phi \cdot \Phi \cdot \Phi+\lim _{R \rightarrow \infty}\left(\int_{V_{f}(R)} \boldsymbol{M}^{T} \cdot \boldsymbol{M} \mathrm{d} V-\frac{9 \pi}{2} \Phi \cdot \Phi R\right)\right\} \cdot \dot{\boldsymbol{U}}_{s}(t) \\
& +\frac{3}{8}\left(\frac{\operatorname{ReS} l}{\pi}\right)^{\frac{1}{2}}\left\{\int _ { - \infty } ^ { t } \left[\frac{2}{3} \boldsymbol{F}_{s}^{H \|}(t)-\left\{\frac{1}{|\boldsymbol{A}|^{2}}\left(\frac{\pi^{\frac{1}{2}}}{2|\boldsymbol{A}|} \operatorname{erf}(|\boldsymbol{A}|)-\exp \left(-|\boldsymbol{A}|^{2}\right)\right)\right\} \boldsymbol{F}_{s}^{H \mid}(s)\right.\right. \\
& \left.+\frac{2}{3} \boldsymbol{F}_{s}^{H \perp}(t)-\left\{\exp \left(-|\boldsymbol{A}|^{2}\right)-\frac{1}{2|\boldsymbol{A}|^{2}}\left(\frac{\pi^{\frac{1}{2}}}{2|\boldsymbol{A}|} \operatorname{erf}(|\boldsymbol{A}|)-\exp \left(-|\boldsymbol{A}|^{2}\right)\right)\right\} \boldsymbol{F}_{s}^{H \perp}(s)\right] \\
& \left.\times \frac{2 \mathrm{~d} s}{(t-s)^{\frac{3}{2}}}\right\} \cdot \Phi \\
& -\operatorname{Re} \lim _{R \rightarrow \infty} \int_{V_{f^{\prime}(R)}}\left(\boldsymbol{u}_{0} \cdot \nabla u_{0}-\boldsymbol{U}_{s}(t) \cdot \nabla u_{0}\right) \cdot \boldsymbol{M} \mathrm{d} V+o(\operatorname{ReSl})+o(\operatorname{Re}) .
\end{aligned}
$$

The first term of this expression is due to an accelerating reference frame, the second is the pseudo-steady Stokes drag, and the third has been labelled as the acceleration reaction. The fourth term represents the unsteady Oseen correction to the hydrodynamic force. It is a new history integral that replaces the Basset history force in the long-time limit at finite Reynolds number. The last term of this expression can only contribute a force perpendicular to the slip velocity of the particle. In order to make use of this expression for a given particle we only require the steady Stokes drag and the corresponding steady Stokes velocity field created by the translating particle.

\section{Results and discussion}

When the timescale is $O\left(v / U_{c}^{2}\right)$, or longer, the history force represented by the unsteady Oseen correction is $O(R e)$ and has a temporal behaviour very different from the Basset term; for shorter timescales it behaves simply as the Basset history force in the long-time limit. This is due to the fact that the timescale must be of this large magnitude for vorticity to have diffused out to the Oseen distance of $a R e^{-1}$. When this has occurred vorticity is swept up in a wake region behind the particle and transported by convection. Also, on this long timescale, the acceleration reaction (the third term of (8.24)) is negligible, being $O\left(R e^{2}\right)$. Owing to the change in the mechanism of vorticity transport - convection in the wake versus radial diffusion we have shown that for timescales greater than $O\left(v / U_{c}^{2}\right)$ the temporal behaviour of the force will decay faster than the $t^{-\frac{1}{2}}$ predicted by unsteady Stokes flow. 
It is important to note that (8.24) is valid for all timescales for the case of a rigid spherical particle. This condition exists because the asymptotic form of the unsteady Stokes force is the same in the limit of very large and very small timescales. The corresponding expression for a spherical particle is given by (7.3). This expression would be appropriate for the calculation to $O(R e)$ of the hydrodynamic force acting on a sphere undergoing any time-dependent motion in a prescribed uniform flow when the particle Reynolds number is small but finite. As demonstrated in the previous section, it can also be used for the dynamic calculation of the trajectory of the particle under the action of an external force.

To reiterate the approach we have used, the analysis begins by constructing a uniformly valid velocity field which is then used in the appropriate reciprocal theorem. This uniformly valid velocity field is constructed by summing the leading-order field valid close to the particle with that valid far from the particle and subtracting the parts common to both fields. The near field is given by the steady (or unsteady) Stokes equations. It is this field which takes into account the finite size and shape of the particle. The far field, when one exists, is given by a point-forced equation with only the dominant inertial terms retained; in the far field the particle appears as a force monopole to leading order. Then the common part is simply the point-forced Stokes (or unsteady Stokes) velocity field.

In performing calculations, the way these fields are distributed is dictated by the necessity to maintain convergent volume integrations. In Appendix B, the outer field, $\boldsymbol{v}_{1}^{p+}$, was formed by subtracting the point-forced unsteady Stokes field so that the Stokeslet nature of the field at the origin was removed. This rearrangement allowed integration to be extended to the centre of the particle and the subsequent application of Fourier transforms and the convolution theorem in $\$ 6$. Had this rearrangement not been performed, the resulting term, $\boldsymbol{U}_{s}(t) \cdot \nabla v_{0}^{p+} \cdot \boldsymbol{M}_{p}$, would have been a conditionally convergent antisymmetric integrand at the origin, being $O\left(|\boldsymbol{r}|^{-3}\right)$. Also, in grouping the velocity fields the way we did, the unsteady Stokes force was retained as a separate contribution, which could be treated on its own.

On the other hand, when the unsteady Stokes force was evaluated in $\S 8$, the outer field was taken as the point-forced unsteady Stokes field. Since it formed a convergent integrand both at the origin and at infinity, this again allowed the application of Fourier transforms and the convolution theorem. In this way the common part, the Stokeslet, was subtracted from the inner steady Stokes field to form the new field given by $\boldsymbol{M}_{s} \cdot \boldsymbol{U}_{s}(t)$. Then once proper account was taken of possible dipole contributions, this field yielded a convergent contribution to the hydrodynamic force when used in the reciprocal theorem. Had the Stokeslet not been subtracted from the inner field, the resulting integrand, $\partial \boldsymbol{u}_{0} / \partial \boldsymbol{t} \cdot \boldsymbol{M}$, would not have been convergent when integrated to infinity, being $O\left(r^{-2}\right)$ at large distances from the particle.

In using this approach to obtain inertial corrections to the steady Stokes force acting on the particle, one is able to attribute the various contributions directly to regions in the fluid domain and the corresponding velocity field. This method contrasts with the usual approach of applying matching conditions and the subsequent evaluation of contributions to the hydrodynamic force from higher-order inner fields. In a sense, the matching procedure is done implicitly by the formation of a uniformly valid velocity field. An advantage of this approach is that, in general, the evaluation of higher-order fields is not required to obtain the leading-order corrections to the steady Stokes force. In addition, only the Fourier space solution to the outer field (B 18) is needed to perform the calculations. As an added comment, we note that the Laplace transform (in time) method of Bentwich \& Miloh (1978) could not be used 
here because of the problem of dealing with the time-dependent coefficient, $U_{s}(t)$, in the convective terms of the outer velocity field.

The equation for the outer contribution to the hydrodynamic force, (6.2), can be shown to be equivalent to previous researchers' findings (Childress 1964; Saffman 1965 ) in that it represents a term proportional to a uniform flow created by the outer velocity field at the centre of the particle. The governing equations for the $\operatorname{Rev}_{1}^{p+}$-field dictate that

$$
\begin{aligned}
\widehat{\boldsymbol{f}_{\text {out }}}\left(\operatorname{Re} \boldsymbol{v}_{1}^{p+}\right)(\boldsymbol{k}) & =\operatorname{Re}\left(\operatorname{ReSl} \frac{\widehat{\partial v_{1}^{p+}}}{\partial t}-2 \pi \mathrm{i} \operatorname{Re} U_{s}(t) \cdot \widehat{k} \widehat{v_{1}^{p+}}\right) \\
& =-4 \pi^{2} \operatorname{Re} k^{2} \widehat{v_{1}^{p+}}-2 \pi \mathrm{i} \operatorname{Re} U_{s}(t) \cdot \boldsymbol{k} \widehat{v^{p}} .
\end{aligned}
$$

Thus, if $(9.1 b)$ is combined with (6.4), the Fourier transform of the $\boldsymbol{M}_{p}$-field, in (6.2), we find

$$
\boldsymbol{F}_{\text {out }}^{H}(t)=6 \pi \Phi \cdot \int_{V_{\infty}}\left(\boldsymbol{R e} \widehat{\boldsymbol{v}_{1}^{++}}-\frac{\mathrm{i} U_{s}(t) \cdot \boldsymbol{k}}{2 \pi k^{2}} \widehat{\boldsymbol{v}^{p}}\right) \mathrm{d} \boldsymbol{k} .
$$

The second term of this expression has no effect except to remove the conditionally convergent antisymmetric contribution that was discussed above. Therefore, (9.2) demonstrates that this contribution is simply the dot product of $6 \pi \Phi$ with the uniform flow created by the disturbance field $R e v_{1}^{p+}$ at the centre of the particle.

The extension of this analysis to include particle rotation does not significantly alter the derivation provided we have

$$
\frac{\left|\Omega_{p}(t)\right| a}{\left|U_{s}(t)\right|} \leqslant O(1)
$$

where $\Omega_{p}(t)$ is the angular velocity of the particle. This condition implies that the Reynolds number based on the characteristic slip velocity is of the same magnitude as that based on the characteristic angular velocity of the particle. The only adjustment for the derivation of the outer contribution from $\$ 6$ is to recognize the fact that $\boldsymbol{F}_{s}^{H}(t)$ is given by

$$
\boldsymbol{F}_{s}^{H}(t)=-6 \pi \Phi(t) \cdot \boldsymbol{U}_{s}(t)-\boldsymbol{R}_{F \Omega}(t) \cdot \Omega_{p}(t),
$$

where $\boldsymbol{R}_{F \Omega}(t)$ is the resistance tensor relating particle rotation to hydrodynamic force. For non-spherical particles both resistance tensors may be time dependent due to the rotation of the particle.

In the presence of particle rotation, the boundary conditions on the surface of the particle must be appropriately modified in the governing equations for the inner velocity fields. With particle rotation the symmetry arguments described in $\S 7$ for a spherical particle no longer hold for the contributions from the regular perturbation to unsteady Stokes flow. These will make an $O(R e)$ contribution in the presence of particle rotation. Thus, the conclusion that the expression given by (7.3) holds for all timescales would no longer be valid. However, for it to be valid to $O(R e)$ and to $O(R e S l)$ when $R e S l \leqslant O(1)$, the only modification required is to include the contribution from the expression given by (8.22). That is,

$$
-R e \lim _{R \rightarrow \infty} \int_{V_{f}(R)}\left(\boldsymbol{u}_{0} \cdot \nabla \boldsymbol{u}_{0}-\boldsymbol{U}_{s}(t) \cdot \nabla \boldsymbol{u}_{0}\right) \cdot \boldsymbol{M} \mathrm{d} V=\pi \operatorname{Re} \Omega_{p}(t) \times \boldsymbol{U}_{s}(t),
$$

where $\Omega_{p}(t)$ has been non-dimensionalized by $U_{c} / a$ and $\times$ indicates a vector cross product. Here, $\boldsymbol{u}_{0}$ is the steady Stokes velocity field created by the sphere translating 
and rotating in a stationary fluid. This represents the lift force first obtained by Rubinow \& Keller (1961). We note that in this derivation the $\boldsymbol{M}$-field remains the same as previously described, i.e. that for particle translation only.

To modify the expression given by $(8.24)$ for non-spherical particle rotation one must first apply the $\boldsymbol{F}_{s}^{H}(t)$ as given by (9.4) where the resistance tensors may also be time dependent. The acceleration reaction contribution, given as the third term of (8.24), requires detailed modifications of the analysis given in $\S 8$. That is, in (8.24) we have assumed $\Phi$ is not a function of time. If, however, the particle was non-spherical and as it translated it was tumbling about an axis that was not an axis of symmetry, $\Phi$ would be time dependent. Under these conditions the acceleration reaction would be replaced by

$$
\begin{aligned}
\operatorname{ReSl}\left\{\lim _{x \rightarrow-\infty}\right. & \frac{1}{\pi}\left[\int_{x}^{t} \ddot{\boldsymbol{F}}_{s}^{H}(q) \cdot \boldsymbol{J}(q, t) \mathrm{d} q+\dot{\boldsymbol{F}}_{s}^{H}(x) \cdot \boldsymbol{J}(x, t)\right] \\
& \left.-\lim _{R \rightarrow \infty} \int_{V_{f}(R)}\left(\frac{\partial \boldsymbol{u}_{0}}{\partial t} \cdot \boldsymbol{M}-\frac{\partial \boldsymbol{u}_{0}^{p}}{\partial t} \cdot \boldsymbol{M}_{p}\right) \mathrm{d} V+\int_{V_{p}} \frac{\partial \boldsymbol{u}_{0}^{p}}{\partial t} \cdot \boldsymbol{M}_{p} \mathrm{~d} V\right\}
\end{aligned}
$$

where

$$
J(x, t)=\int_{x}^{t} \frac{\Phi(s)}{((t-s)(s-x))^{\frac{1}{2}}} \mathrm{~d} s \cdot \Phi(t) .
$$

When $\Phi$ is independent of time, $\boldsymbol{J}(x, t)$ is equal to $\pi \Phi \cdot \Phi$. In the above expression, as well as in the last term of (8.24), the steady Stokes velocity field for the particle both translating and rotating in a stationary fluid is required for the $\boldsymbol{u}_{0}$-field. It should also be noted that the Stokes velocity fields $\boldsymbol{u}_{0}$ and $\boldsymbol{u}_{0}^{p}$ are in general functions of time not only because of the time-dependent nature of $\boldsymbol{U}_{s}(t)$ and $\Omega_{p}(t)$ but also because of the change in orientation of the particle with time. For example, if $\boldsymbol{\mu}_{0}=\boldsymbol{M} \cdot \boldsymbol{U}_{\mathrm{s}}(t)$, then $\boldsymbol{M}$ may also be a function of time through the particle orientation dependence relative to $U_{s}(t)$. As a final note, the second-order tensor $\Phi$ in these modified expressions remains as defined above since the $\boldsymbol{M}$-field is expressed in terms of the particle translating in the given orientation at time $t$.

Another possibility for extension of these results would be to consider the case of unsteady particle motion in a linear flow. Maxey \& Riley (1983) have obtained a solution for the corresponding unsteady Stokes problem associated with a translating sphere in a time-dependent non-uniform flow. Their analysis shows there is no significant modification of the unsteady Stokes force from that of (1.2) for the case of a linear flow. The only difference is that the particle velocity is given as relative to the undisturbed flow evaluated at the particle centre. It is believed that the inclusion of the convective inertial corrections would be of more significance in this case than in the uniform flow problem analysed here, because the critical timescale for which an outer region develops, where the convective inertia is important to leading order, is $\dot{\gamma}^{-1}$, where $\dot{\gamma}$ is the characteristic strain rate of the external flow. This is a more relevant timescale since it does not depend on the fluid viscosity nor on any external forces that act on the particle. The dominant convective correction to the unsteady Stokes force would in general come from the outer contribution since it is $O\left(\operatorname{Re}_{\hat{\gamma}}^{\frac{1}{2}}\right)$ where $R e_{\dot{\gamma}}=a^{2} \dot{\gamma} / v$ (Saffman 1965). This correction could be solved for, in principle, by the analysis presented here, but it would ultimately require the solution of a partial differential equation for the velocity field in Fourier space.

As a final note, the present approach has also been used to study oscillatory motion 
and the motion of bubbles and drops at small Reynolds number (Lovalenti \& Brady $1993 a, b)$.

P. M. L. wishes to thank Rockwell International for providing financial support as a Rockwell Fellow during the course of this study. The authors also gratefully acknowledge H.A. Stone for many helpful discussions and insightful comments in the preparation of this article.

\title{
Appendix A. The reciprocal theorem and expressions for the particle force
}

\author{
By P. M. Lovalenti, J. F. Brady and Howard A. Stone ${ }^{1}$ \\ ${ }^{1}$ Division of Applied Sciences, Harvard University, Cambridge, MA 02138, USA
}

It is necessary to briefly consider the governing equations and general features of the fluid motion about a rigid particle translating with velocity $U_{p}$ in a general time-dependent flow. Relative to a coordinate system fixed in the laboratory, the undisturbed fluid motion is denoted $v^{\infty}(x, t)$. Referred to a coordinate system instantaneously fixed to the particle, the undisturbed fluid motion is denoted $\boldsymbol{u}^{\infty}(\boldsymbol{r}, t)$, where $\boldsymbol{v}^{\infty}(\boldsymbol{x}, t)=\boldsymbol{U}^{\infty}+\boldsymbol{u}^{\infty}(\boldsymbol{r}, t)$ and $\boldsymbol{U}^{\infty}=\boldsymbol{v}^{\infty}\left(\boldsymbol{Y}_{p}(t), t\right)$ is the fluid velocity evaluated at the current particle location.

The disturbance flow equations relative to a particle-fixed observer enter frequently in the analysis below. Denoting the disturbance velocity, pressure, and stress by $(u, p, \sigma)$, the disturbance flow satisfies

$$
\begin{aligned}
\nabla^{2} \boldsymbol{u}-\nabla p=\nabla \cdot \boldsymbol{\sigma} & =\operatorname{Re}\left[S l \frac{\partial \boldsymbol{u}}{\partial t}+\boldsymbol{u} \cdot \boldsymbol{\nabla u}+\boldsymbol{u} \cdot \boldsymbol{\nabla} \boldsymbol{u}^{\infty}+\boldsymbol{u}^{\infty} \cdot \boldsymbol{\nabla u}\right. \\
& \left.+\left(U^{\infty}-U_{p}\right) \cdot \nabla \boldsymbol{u}\right] \\
& =\boldsymbol{f}\left(\boldsymbol{u}, \boldsymbol{u}^{\infty}\right),
\end{aligned}
$$

and

$$
\boldsymbol{\nabla} \cdot \boldsymbol{u}=0
$$

where we have assumed $v^{\infty}$ satisfies the Navier-Stokes equations. The function $f$ will be used throughout to represent the inertial terms in the equations of motion. This term clearly depends on the details of the undisturbed fluid motion and the disturbance flow generated by the particle. Also, the disturbance flow problem satisfies the boundary conditions

$$
\boldsymbol{u}=\boldsymbol{U}_{p}-\boldsymbol{v}^{\infty}(\boldsymbol{x}, t) \quad \text { for } \boldsymbol{r}=\boldsymbol{x}-\boldsymbol{Y}_{p}(t) \in S_{p}
$$

and

$$
\boldsymbol{u}, p \rightarrow 0 \quad \text { as } r=|\boldsymbol{r}| \rightarrow \infty \text {. }
$$

Notice that the disturbance velocity field $\boldsymbol{u}(\boldsymbol{r}, t)$ describes fluid motion in the vicinity of the particle relative to an observer moving along the streamline, which, at time $t$, passes through the particle centre. The final term in equation (A $1 a$ ) represents a slip between the actual particle velocity and the undisturbed flow evaluated at the instantaneous location of the particle.

Translational accelerations of the particle arise to balance the difference between 
the hydrodynamic and external forces, $\boldsymbol{F}^{\text {ext }}$. The hydrodynamic force $\boldsymbol{F}^{H}$ exerted by the fluid on the particle is given by

$$
\operatorname{ReSl} \widetilde{m}_{p} \dot{U}_{p}-\boldsymbol{F}^{e x t}=\int_{S_{p}} \boldsymbol{n} \cdot \boldsymbol{\sigma}_{T} \mathrm{~d} S=\boldsymbol{F}^{H},
$$

where $\widetilde{m}_{p}$ is the mass of the particle non-dimensionalized by the product of the density of the fluid, $\rho$, and the cube of the characteristic particle dimension, $a^{3}$. Here, $\sigma_{T}$ denotes the stress tensor for the actual flow about the particle.

We now outline the use of the reciprocal theorem to derive a general expression, including inertial effects, for the hydrodynamic force as a function of the timedependent undisturbed velocity field. This application of the reciprocal theorem is similar to the procedure used by previous investigators (Cox \& Brenner 1968; Ho \& Leal 1974; Leal 1980). These earlier authors were interested in calculating the inertial contribution to particle translational and rotational velocities for situations where the Reynolds number was sufficiently small that a rigid boundary was reached prior to an $O\left(R e^{-\frac{1}{2}}\right)$ distance where fluid inertia becomes significant. Consequently, the boundary was located in the 'inner' region and a regular perturbation method accounting for inertial effects was applicable. Contrary to statements made in earlier studies, however, here we show that it is possible to use the reciprocal theorem in those instances where fluid inertia enters directly and, in principle, a solution for the detailed flow field would require singular perturbation methods. In this case, we require that the 'outer' region where fluid inertia becomes significant is closer to the particle than any boundaries; i.e. $O\left(\operatorname{Re}^{-\beta}\right)<L / a$, where $L$ is a representative geometric length scale and where, for example, $\beta=1$ for uniform flow and $\beta=1 / 2$ for linear flow.

Consider two solutions of the Navier-Stokes equations $(\boldsymbol{u}, \boldsymbol{\sigma}, \boldsymbol{f})$ and $(\hat{\boldsymbol{u}}, \hat{\boldsymbol{\sigma}}, \hat{\boldsymbol{f}})$ where $\boldsymbol{f}$ and $\hat{\boldsymbol{f}}$ represent the inertial (steady and unsteady) terms. Also, $\nabla \cdot \boldsymbol{u}=0$ and $\nabla \cdot \hat{\boldsymbol{u}}=0$. The reciprocal theorem states that at any time $t$,

$$
\int_{S}(\boldsymbol{n} \cdot \boldsymbol{\sigma}) \cdot \hat{\boldsymbol{u}} \mathrm{d} S+\int_{V_{f}} \boldsymbol{f} \cdot \hat{\boldsymbol{u}} \mathrm{d} V=\int_{S}(\boldsymbol{n} \cdot \hat{\boldsymbol{\sigma}}) \cdot \boldsymbol{u} \mathrm{d} S+\int_{V_{f}} \hat{\boldsymbol{f}} \cdot \boldsymbol{u} \mathrm{d} V
$$

where $S$ represents all bounding surfaces, $S=S_{p}+S_{\infty}$, and $n$ is the unit outward normal directed from $S$ into the fluid volume $V_{f}$. We will neglect the presence of any walls since here we are concerned with an isolated particle in an unbounded prescribed flow.

Let the unhatted flow correspond to the disturbance flow problem defined earlier by equation (A 1). Let $(\hat{\boldsymbol{u}}, \hat{\boldsymbol{\sigma}}, \hat{\boldsymbol{f}})$ correspond to the Stokes flow problem of a rigid particle translating with velocity $\hat{U}$ :

$$
\nabla \cdot \hat{u}=0, \quad \nabla \cdot \hat{\sigma}=0, \quad \hat{\boldsymbol{f}}=0
$$

with

$$
\hat{\boldsymbol{u}}=\hat{\boldsymbol{U}} \quad \text { for } \boldsymbol{r} \in S_{p} \quad \text { and } \hat{\boldsymbol{u}} \rightarrow 0 \quad \text { as } \quad|\boldsymbol{r}| \rightarrow \infty .
$$

Owing to the linearity of the governing equations and boundary conditions, the solution of this fundamental Stokes flow problem will take the form

$$
\hat{\boldsymbol{u}}(\boldsymbol{r})=\boldsymbol{M} \cdot \hat{\boldsymbol{U}}
$$

where the second-rank tensor $\boldsymbol{M}$ is a function of position; for a spherical particle it 
has the well-known form

$$
M=\frac{3}{4}\left(\frac{1}{r}+\frac{r r}{r^{3}}\right)+\frac{1}{4}\left(\frac{1}{r^{3}}-\frac{3 r r}{r^{5}}\right) .
$$

The disturbance Stokes flow problem has the familiar properties

$$
|\hat{\boldsymbol{u}}| \sim O(1 / r) \quad \text { as } r \rightarrow \infty, \quad|\hat{\boldsymbol{\sigma}}| \sim O\left(1 / r^{2}\right) \quad \text { as } r \rightarrow \infty .
$$

Although (A 1) cannot be solved exactly (unless $R e \equiv 0$ ), nevertheless for any Reynolds number it is clear that for large $r$

$$
|\boldsymbol{u}| \sim O\left(r^{-\alpha}\right), \quad \text { with } \alpha>0 .
$$

Hence, the integral over $S_{\infty}$ in (A 4) involving $(\boldsymbol{n} \cdot \hat{\boldsymbol{\sigma}}) \cdot \boldsymbol{u}$ will vanish as the surface $S_{\infty}$ moves off to infinity. Similarly, the viscous stress associated with $\sigma$ will scale as $|\nabla u| \sim O\left(r^{-\alpha-1}\right)$, and thus this part of $(\boldsymbol{n} \cdot \boldsymbol{\sigma}) \cdot \hat{\boldsymbol{u}}$ on $S_{\infty}$ will also give zero. Thus, we are left to consider the integral

$$
-\int_{S_{\infty}} p \boldsymbol{n} \cdot \hat{\boldsymbol{u}} \mathrm{d} S .
$$

If in the far field viscous terms are larger than or the same order as inertial terms, then $p$ will also scale as $r^{-\alpha-1}$ and this integral will not contribute. If there are regions in the outer flow that are inviscid, then either these regions will be irrotational or rotational and the pressure must be estimated from Bernoulli's equation. In this case it is not obvious that $p$ decays sufficiently rapidly to neglect this integral, although it seems most likely that this will be the case.

For the calculations performed in this paper, which are perturbations about the zero-Reynolds-number solution, $p$ scales viscously and we may safely neglect all terms which involve $S_{\infty}$. Thus, the reciprocal theorem (A 4) may now be written in the simpler 'disturbance' form

$$
\int_{S_{p}}(\boldsymbol{n} \cdot \boldsymbol{\sigma}) \cdot \hat{\boldsymbol{u}} \mathrm{d} S+\int_{V_{f}} \boldsymbol{f} \cdot \hat{\boldsymbol{u}} \mathrm{d} V=\int_{S_{p}}(\boldsymbol{n} \cdot \hat{\boldsymbol{\sigma}}) \cdot \boldsymbol{u} \mathrm{d} \boldsymbol{S},
$$

which, with the caveat about inviscid flow, is an exact statement valid at any time $t$ for an isolated rigid particle in a prescribed unbounded flow at arbitrary particle Reynolds number $R e$.

We will now make use of the definition of the disturbance flow problems $u$, which is the flow involving inertia, and $\hat{u}$, which is the fundamental solution to typical Stokes flow problems, in order to derive a general equation relating the hydrodynamic force on the particle, to the undisturbed fluid motion $v^{\infty}(x, t)$.

Using (A $5 b$ ) for the boundary condition on $\hat{u}$, it follows that

$$
\int_{S_{p}}(\boldsymbol{n} \cdot \boldsymbol{\sigma}) \cdot \hat{\boldsymbol{u}} \mathrm{d} S=\int_{\mathrm{S}_{p}} \boldsymbol{n} \cdot \boldsymbol{\sigma} \mathrm{d} \boldsymbol{S} \cdot \hat{\boldsymbol{U}} .
$$

Now it only remains to identify the disturbance flow stress as $\sigma=\sigma_{T}-\sigma^{\infty}$, where $\sigma_{T}$ is the stress tensor for the actual flow about the particle, $\sigma^{\infty}$ is the stress tensor for the undisturbed motion, and so involves $v^{\infty}$, and $\sigma$ is defined by the disturbance flow problem (A 1). It follows that the term on the right-hand side of (A 11) is related to the particle force (see (A 3)). Thus, (A 11) becomes

$$
\int_{S_{p}}(\boldsymbol{n} \cdot \boldsymbol{\sigma}) \cdot \hat{\boldsymbol{u}} \mathrm{d} S=\boldsymbol{F}^{H} \cdot \hat{\boldsymbol{U}}-\int_{S_{p}}\left(\boldsymbol{n} \cdot \boldsymbol{\sigma}^{\infty}\right) \mathrm{d} S \cdot \hat{\boldsymbol{U}}
$$


Applying the divergence theorem to the integral involving the undisturbed flow, we obtain

$$
\int_{S_{p}}(\boldsymbol{n} \cdot \boldsymbol{\sigma}) \cdot \hat{u} \mathrm{~d} S=\boldsymbol{F}^{H} \cdot \hat{\boldsymbol{U}}-\int_{V_{p}}\left(\boldsymbol{\nabla} \cdot \boldsymbol{\sigma}^{\infty}\right) \mathrm{d} V \cdot \hat{\boldsymbol{U}} .
$$

The evaluation of this integral involving the undisturbed motion is most easily performed by referring all velocities to the laboratory frame. In this case $\boldsymbol{v}^{\infty}(\boldsymbol{x}, t)$ satisfies

where

$$
\nabla \cdot \sigma^{\infty}=\operatorname{Re} \frac{\mathrm{D}^{\infty} \boldsymbol{v}^{\infty}}{\mathrm{D} t}
$$

$$
\frac{\mathrm{D}^{\infty}}{\mathrm{D} t} \equiv S l \frac{\partial}{\partial t}+\boldsymbol{v}^{\infty}(\boldsymbol{x}, t) \cdot \nabla
$$

The right-hand side of (A $14 a$ ) is a function of position $x$ and when substituted in (A 13) yields

$$
\int_{S_{p}}(\boldsymbol{n} \cdot \boldsymbol{\sigma}) \cdot \hat{\boldsymbol{u}} \mathrm{d} S=\boldsymbol{F}^{H} \cdot \hat{\boldsymbol{U}}-\operatorname{Re} \int_{V_{p}} \frac{\mathrm{D}^{\infty} \boldsymbol{v}^{\infty}}{\mathrm{D} t} \mathrm{~d} \boldsymbol{V} \cdot \hat{\boldsymbol{U}}
$$

Next consider the integral on the right-hand side of (A 10). Using the boundary condition on the surface of the particle associated with the disturbance flow problem (A $2 a$ ), we have

$$
\int_{S_{p}}(\boldsymbol{n} \cdot \hat{\boldsymbol{\sigma}}) \cdot \boldsymbol{u} \mathrm{d} S=\int_{S_{p}} \boldsymbol{n} \cdot \hat{\boldsymbol{\sigma}} \mathrm{d} S \cdot \boldsymbol{U}_{p}-\int_{S_{p}}(\boldsymbol{n} \cdot \hat{\boldsymbol{\sigma}}) \cdot \boldsymbol{v}^{\infty} \mathrm{d} S
$$

or

$$
\int_{S_{p}}(\boldsymbol{n} \cdot \hat{\boldsymbol{\sigma}}) \cdot \boldsymbol{u} \mathrm{d} S=\hat{\boldsymbol{F}} \cdot \boldsymbol{U}_{p}-\int_{S_{\mathrm{p}}}(\boldsymbol{n} \cdot \hat{\boldsymbol{\sigma}}) \cdot \boldsymbol{v}^{\infty} \mathrm{d} S,
$$

where $\hat{\boldsymbol{F}}$ is the force acting on the particle associated with the disturbance Stokes flow problem.

To proceed further to simplify (A 17) one can either use a known solution for the Stokes flow about the particle or one must have knowledge of the nature of the undisturbed motion and appeal to the symmetry properties of the resistance tensors associated with the particle force. We pursue the first approach now by considering the flow about a spherical particle. We will return to the second approach later for the case when the undisturbed flow is uniform. by

On the surface of a sphere, the force per unit area in Stokes flow is given simply

$$
\left.\boldsymbol{n} \cdot \hat{\boldsymbol{\sigma}}_{T}\right|_{r=1, r=n}=-\frac{3}{2} \hat{\boldsymbol{U}}
$$

Substituting (A 18) into the integral on the right-hand side of (A 17) yields

$$
\int_{S_{p}}(\boldsymbol{n} \cdot \hat{\boldsymbol{\sigma}}) \cdot \boldsymbol{u} \mathrm{d} S=\left[-6 \pi \boldsymbol{U}_{p}+\frac{3}{2} \int_{S_{p}} \boldsymbol{v}^{\infty} \mathrm{d} S\right] \cdot \hat{\boldsymbol{U}} .
$$

Here we have used the known result for a sphere that $\hat{\boldsymbol{F}}=-6 \pi \hat{\boldsymbol{U}}$. Eliminating $\hat{\boldsymbol{U}}$ from the problem, we find that when (A 15) and (A 19) are combined in the reciprocal theorem (A 10) they yield the following relationship for a sphere:

$$
\boldsymbol{F}^{H}-\boldsymbol{R} \boldsymbol{e} \int_{V_{p}} \frac{\mathrm{D}^{\infty} \boldsymbol{v}^{\infty}}{\mathrm{D} t} \mathrm{~d} V=-6 \pi \boldsymbol{U}_{p}+\frac{3}{2} \int_{S_{p}} \boldsymbol{v}^{\infty} \mathrm{d} S-\int_{V_{f}} \boldsymbol{f} \cdot \boldsymbol{M} \mathrm{d} \boldsymbol{V} .
$$


Each of the integrals involving the undisturbed motion in equation (A 20) can be evaluated by using a Taylor series expansion about the instantaneous centre of the particle $\boldsymbol{Y}_{p}(t)$ (assuming the velocity gradient variations are small over the length scale of the particle) and performing the integrations; here, quadratic variations are retained. Using (A 3), which balances the particle translational acceleration against the net forces, leads to the general expression for a sphere:

$$
\begin{aligned}
\frac{4 \pi}{3} \operatorname{Re} & {\left[\frac{\rho_{p}}{\rho_{f}} \dot{\boldsymbol{U}}_{p}-\left(\frac{\mathrm{D}^{\infty} \boldsymbol{v}^{\infty}}{\mathrm{D} t}\right)_{\boldsymbol{Y}_{p}(t)}-\frac{1}{10} \nabla^{2}\left(\frac{\mathrm{D}^{\infty} \boldsymbol{v}^{\infty}}{\mathrm{D} t}\right)_{\boldsymbol{Y}_{p}(t)}\right] } \\
& =\boldsymbol{F}^{e x t}-6 \pi\left[\boldsymbol{U}_{p}-\boldsymbol{U}^{\infty}-\frac{1}{6} \nabla^{2} \boldsymbol{v}^{\infty}\left(\boldsymbol{Y}_{p}(t), t\right)\right]-\int_{V_{f}} \boldsymbol{f} \cdot \boldsymbol{M} \mathrm{d} \boldsymbol{V},
\end{aligned}
$$

where $\rho_{p}$ is the density of the particle. In principle, equation (A 21) is valid for any Reynolds number $R e$ at any time $t$. Specifically, we have found this formulation useful for an examination of inertial corrections at low Reynolds numbers. It is a generalization incorporating all inertial influences of a result of Maxey \& Riley (1983), which was restricted to the unsteady Stokes equations.

Now for a non-spherical particle in a uniform undisturbed flow $\left(v^{\infty} \equiv U^{\infty}(t), u^{\infty}=0\right)$ (A 17) becomes

$$
\int_{S_{p}}(\boldsymbol{n} \cdot \hat{\boldsymbol{\sigma}}) \cdot \boldsymbol{u} \mathrm{d} S=\hat{\boldsymbol{F}} \cdot\left(\boldsymbol{U}_{p}-\boldsymbol{U}^{\infty}\right)
$$

Using the resistance tensor form for Stokes flow, $\hat{\boldsymbol{F}}$ may be expressed as

$$
\hat{\boldsymbol{F}}=-\boldsymbol{R}_{F U} \cdot \hat{\boldsymbol{U}}
$$

where $\boldsymbol{R}_{F U}$ is the second-rank tensor relating particle velocity to the drag. Since $\boldsymbol{R}_{F U}$ is a symmetric tensor, (A 22) is now given by

$$
\int_{S_{p}}(\boldsymbol{n} \cdot \hat{\boldsymbol{\sigma}}) \cdot \boldsymbol{u} \mathrm{d} S=\boldsymbol{F}_{s}^{H} \cdot \hat{\boldsymbol{U}}
$$

where $\boldsymbol{F}_{s}^{H}\left(=-\boldsymbol{R}_{F U} \cdot\left(\boldsymbol{U}_{p}-\boldsymbol{U}^{\infty}\right)\right)$ is the actual Stokes drag acting on the particle. If we combine (A 15) with (A 24) in the reciprocal theorem (A 10) and eliminate $\hat{U}$, we find the following expression for the hydrodynamic force acting on an arbitrarily shaped particle translating in a uniform flow:

$$
\boldsymbol{F}^{H}(t)=\boldsymbol{F}_{s}^{H}(t)+\operatorname{ReSl} \widetilde{V}_{p} \dot{\mathbf{U}}^{\infty}(t)-\int_{V_{f}} \boldsymbol{f} \cdot \boldsymbol{M} \mathrm{d} V .
$$

Here, $\widetilde{V}_{p}$ is the volume of particle non-dimensionalized by $\boldsymbol{a}^{3}$ and $\boldsymbol{M} \cdot \hat{\boldsymbol{U}}$ is the Stokes velocity field caused by the particle translating with velocity $\hat{U}$.

As a final note, analogous expressions for the hydrodynamic torque and particle stresslet have also been derived with this approach by introducing a rotational velocity and rate-of-strain tensor, respectively, into the surface boundary condition (A $5 b$ ) for the disturbance Stokes flow problem (Stone \& Brady 1993).

\section{Appendix B. Scaling arguments for the uniformly valid velocity field}

We identify three cases to be considered based on the relative magnitude of $R e$ and $R e S l: R e S l \gg O\left(R e^{2}\right), R e S l=O\left(R e^{2}\right)$, and $R e S l \ll O\left(R e^{2}\right)$. In each case, we attempt to rescale length to find if a dominant balance exists in the governing equation such 
that convective inertia is retained to leading order. That is, we wish to find 'outer' velocity fields where convective inertial terms are of equal magnitude to viscous or unsteady inertial terms. In all cases the pressure will be rescaled so that it remains to satisfy continuity. Also, in order to obtain further scaling information for the outer velocity fields, we shall employ the method used by Saffman (1965) in replacing the boundary conditions on the surface of the particle with force monopoles, dipoles, etc. at the particle centre.

We shall be considering various velocity fields associated with different governing equations, and each field will have different dependent variables. The general convention for the pressure and velocity symbols are as follows: $(a)(\Pi, v)$ are for unsteady fields, while $(p, \boldsymbol{u})$ for steady fields; $(b)$ a subscript 0 denotes a leading-order field, while a subscript 1 denotes a corresponding velocity field contribution due solely to the convective inertia of the fluid, being identically zero for zero Reynolds number; (c) a superscript $p$ denotes a monopole, or point-force field; $(d)$ a superscript + signifies that a convective inertial term is retained in the governing equation for the leading-order field. Note that the unsub-(or unsuper-)scripted $(p, \boldsymbol{u})$ will continue to represent the solution to the full Navier-Stokes problem presented in $\$ 2$.

\section{B.1. $S l \gg R e$}

When $S l \gg R e$, or the characteristic time $\tau_{c} \ll v / U_{c}^{2}$, there is no rescaling of the governing equations for which the convective terms can be the same order as the viscous or unsteady terms. This reflects the fact that vorticity has not diffused out to the Oseen distance $a R e^{-1}$. Thus, a uniformly valid first approximation to the velocity field under these conditions will always be given by the unsteady Stokes equations

$$
\begin{gathered}
-\nabla \Pi_{0}+\nabla^{2} \boldsymbol{v}_{0}=\operatorname{ReSl} \frac{\partial \boldsymbol{v}_{0}}{\partial t}, \\
\boldsymbol{\nabla} \cdot \boldsymbol{v}_{0}=0,
\end{gathered}
$$

with

$$
\begin{gathered}
\boldsymbol{v}_{0}=\boldsymbol{U}_{s}(t) \quad \text { on the surface of the particle, } \\
\qquad \boldsymbol{v}_{0}, \Pi_{0} \rightarrow 0 \quad \text { as } \quad|\boldsymbol{r}| \rightarrow \infty .
\end{gathered}
$$

The contribution from the convective inertia will then be simply an $O(R e)$ regular perturbation to unsteady Stokes flow. That is, if we take $\left(\Pi_{0}, v_{0}\right)$ as the solution to the above unsteady Stokes equations, then the $O(R e)$ contribution to the velocity field due to convection, $\left(\operatorname{Re} \Pi_{1}, \operatorname{Re} \boldsymbol{v}_{1}\right)$, must satisfy

$$
\begin{gathered}
-\nabla \Pi_{1}+\nabla^{2} \boldsymbol{v}_{1}-\operatorname{ReSl} \frac{\partial \boldsymbol{v}_{1}}{\partial t}=\boldsymbol{v}_{0} \cdot \boldsymbol{\nabla} \boldsymbol{v}_{0}-\boldsymbol{U}_{s}(t) \cdot \boldsymbol{\nabla} \boldsymbol{v}_{0}, \\
\boldsymbol{\nabla} \cdot \boldsymbol{v}_{1}=0, \\
\boldsymbol{v}_{1}=0 \quad \text { on the surface of the particle, } \\
\boldsymbol{v}_{1}, \Pi_{1} \rightarrow 0 \quad \text { as }|\boldsymbol{r}| \rightarrow \infty .
\end{gathered}
$$

Although this correction is $O(R e)$ and therefore one would expect that when using the reciprocal theorem (3.1) this field could be neglected, it is needed in order to get the proper $O(R e)$ correction to the unsteady term, $R e S l \partial u / \partial t$, in (3.2).

Other than the requirement that $R e S l \gg O\left(\operatorname{Re}^{2}\right)$, the above arguments are for arbitrary magnitudes of ReSl. If, however, we have the additional condition that 
$\operatorname{ReSl} \ll O(1)$, which is equivalent to having a timescale much longer than the diffusive scale $a^{2} / v$, one can apply a singular perturbation analysis to the solution of the unsteady Stokes equations. This is done in $\S 8$ in the main text for the case of non-spherical particles. We only note here that if $\operatorname{Re} S l \ll O(1)$, the governing equations for $\boldsymbol{v}_{0}$ near the particle will be to leading order the steady Stokes equations:

$$
\begin{gathered}
-\nabla p_{0}+\nabla^{2} \boldsymbol{u}_{0}=0, \\
\nabla \cdot \boldsymbol{u}_{0}=0, \\
\boldsymbol{u}_{0}=\boldsymbol{U}_{s}(t) \quad \text { on the surface of the particle, } \\
\boldsymbol{u}_{0}, p_{0} \rightarrow 0 \quad \text { as } \quad|\boldsymbol{r}| \rightarrow \infty .
\end{gathered}
$$

While far from the particle, at distances of $O\left(a(\operatorname{ReSl})^{-\frac{1}{2}}\right)$, they will be given to leading order by point-forced unsteady Stokes equations:

$$
\begin{gathered}
-\nabla \Pi_{0}^{p}+\nabla^{2} v_{0}^{p}=\operatorname{ReSl} \frac{\partial v_{0}^{p}}{\partial t}+\boldsymbol{F}_{s}^{H}(t) \delta(\boldsymbol{r}), \\
\nabla \cdot \boldsymbol{v}_{0}^{p}=0,
\end{gathered}
$$

where $\boldsymbol{F}_{s}^{H}(t)$ is the dimensionless pseudo-steady Stokes drag acting on the particle and $\delta(\boldsymbol{r})$ is the three-dimensional Dirac delta function. Convection will enter as a regular perturbation to these fields in their respective regions of validity.

$$
\text { B.2. } S l=O(R e)
$$

When $S l=O(R e)$, there is a dominant balance between the viscous and both the unsteady and convective inertial terms. This signals the existence of a singular perturbation expansion in small $R e$. The timescale corresponding to this condition is $\tau_{c} \approx v / U_{c}^{2}$, and was also identified by Bentwich \& Miloh (1978) in their analysis. If we define outer variables as

$$
\overline{\boldsymbol{r}}=\operatorname{Rer}, \quad \bar{p}=R e^{-2} p, \quad \overline{\boldsymbol{u}}=R e^{-1} \boldsymbol{u},
$$

the full governing equations for this 'outer' region become

$$
\begin{aligned}
&-\bar{\nabla} \bar{p}+\bar{\nabla}^{2} \overline{\boldsymbol{u}}= \frac{\partial \overline{\boldsymbol{u}}}{\partial t}-\boldsymbol{U}_{s}(t) \cdot \bar{\nabla} \overline{\boldsymbol{u}}+\boldsymbol{F}_{1}(t) \delta(\overline{\boldsymbol{r}}) \\
&+\operatorname{Re} \overline{\boldsymbol{u}} \cdot \bar{\nabla} \overline{\boldsymbol{u}}+\operatorname{Re}_{2}(t) \cdot \bar{\nabla} \delta(\overline{\boldsymbol{r}})+\ldots, \\
& \bar{\nabla} \cdot \overline{\boldsymbol{u}}=0 .
\end{aligned}
$$

Here, we have replaced the boundary condition on the surface of the particle by a series of multipoles. The first multipole $F_{1}(t)$ is given by

$$
\boldsymbol{F}_{1}(t)=\boldsymbol{F}^{H}(t)-\operatorname{ReSl} \widetilde{V}_{p} \dot{U}_{p}(t),
$$

where $\boldsymbol{F}^{H}(t)$ is the full hydrodynamic force acting on the particle, non-dimensionalized by $\mu a U_{c}$. The volume of the particle $\widetilde{V}_{p}$ has been non-dimensionalized by $a^{3}$. In this case, $\boldsymbol{F}_{1}(t)$ can be approximated by the pseudo-steady Stokes drag, $\boldsymbol{F}_{s}^{H}(t)$, to leading order. The second multipole $F_{2}(t)$ is a second-order tensor which is given by the stresslet and rotlet acting on the particle. The remaining terms (not shown) represent the higher-order multipoles which are of lower-order in Re. Thus, our leading-order outer equations when $R e S l=O\left(R e^{2}\right)$ are given, in the originally scaled variables, by 


$$
\begin{gathered}
-\nabla \Pi_{0}^{p+}+\nabla^{2} v_{0}^{p+}=\operatorname{ReS} l \frac{\partial v_{0}^{p+}}{\partial t}-\operatorname{Re} U_{s}(t) \cdot \nabla v_{0}^{p+}+F_{s}^{H}(t) \delta(r), \\
\nabla \cdot v_{0}^{p+}=0 .
\end{gathered}
$$

The governing equations for the inner region (i.e. for $\left.|r|<O\left(R e^{-1}\right)\right)$ are the steady Stokes equations given by (B 3).

\section{B.3. $S l \ll R e$}

When $S l \ll R e$, or $\tau_{c} \gg v / U_{c}^{2}$, again, to leading order, the velocity field near to the particle is given by the steady Stokes equations. There are two possibilities, however, for producing a balance of terms through rescaling that includes convective and viscous terms. The first results from the rescaling given by (B 5), which yields the steady Oseen equations to leading order:

$$
-\nabla p_{0}^{+}+\nabla^{2} \boldsymbol{u}_{0}^{+}=-\operatorname{Re} \boldsymbol{U}_{s}(t) \cdot \nabla \boldsymbol{u}_{0}^{+}+\boldsymbol{F}_{s}^{H}(t) \delta(\boldsymbol{r}), \quad \nabla \cdot \boldsymbol{u}_{0}^{+}=0 .
$$

The other possibility comes from a balance of the unsteady and convective inertial terms with viscous terms in a region that is $O\left(a S l^{-1}\right)$ from the particle.

To discover this second outer region, the length in the direction of $\boldsymbol{U}_{s}(t)$, considered constant on this long timescale, is rescaled to balance the unsteady and convective inertial terms. Then, the other two mutually orthogonal directions are rescaled to retain diffusive (or viscous) terms, i.e. the classic scalings for an unsteady laminar wake. The pressure gradient scale is determined by using these length scales in the appropriate expression for the pressure created by a Stokeslet (i.e. $\boldsymbol{F}_{s}^{H} \cdot \boldsymbol{x} / 4 \pi r^{3}$ ), as the pressure variation takes this form to leading order in the outer region. Finally, the velocity scales are determined by balancing dominant terms with the pressure gradient in the individual component momentum equations. For example, if we take the $z$-direction as the direction of $U_{s}(t)$, then the $z$-momentum equation becomes to leading order

$$
-\frac{\partial \bar{p}}{\partial \bar{z}}+\left(\frac{\partial^{2}}{\partial \bar{x}^{2}}+\frac{\partial^{2}}{\partial \bar{y}^{2}}\right) \bar{u}_{z}=\left(\frac{\partial}{\partial t}-U_{s} \frac{\partial}{\partial \bar{z}}\right) \bar{u}_{z} .
$$

The rescaling in this equation is given by

$$
(x, y)=(\operatorname{ReS} l)^{-\frac{1}{2}}(\bar{x}, \bar{y}), \quad z=S l^{-1} \bar{z}
$$

and

$$
\frac{\partial p}{\partial z}=S l^{3} \frac{\partial \bar{p}}{\partial \bar{z}}, \quad u_{z}=\frac{S l^{2}}{R e} \bar{u}_{z} .
$$

The length scales associated with these equations are larger than those in the steady Oseen equations above, $O\left(a S l^{-1}\right)$ vs. $O\left(a R e^{-1}\right)$. Thus they are associated with a farther field than the Oseen region which applies to the wake immediately behind the particle. As seen in $\$ 4$, the inclusion of the transient term in the governing equation for this new region of the flow has consequence for the temporal decay of the drag.

The above scaling analysis has identified the form of the outer region depending on the relative magnitudes of $R e S l$ and $R e^{2}$. With this information we now proceed to construct a velocity field that is uniformly valid in space for all times. This velocity field will be used in the volume integral over the entire fluid region when computing the inertial correction to the steady Stokes drag through the reciprocal theorem (3.1).

The field given by the unsteady Stokes equations, $v_{0}$, is uniformly valid for short times, $R e S l \gg O\left(R e^{2}\right)$, and we can add to this the $O(R e)$ regular perturbation, $v_{1}$, to 
include convective effects; specifically, to get the $O(R e)$ correction to $\partial u / \partial t$. Our uniformly valid velocity field for short timescales is then

$$
\boldsymbol{u}_{s}^{u v}=\boldsymbol{v}_{0}+\operatorname{Re} \boldsymbol{v}_{1},
$$

where $v_{0}$ and $v_{1}$ are given by solutions to (B 1$)$ and (B 2), respectively.

For long times when $R e S l \leqslant O\left(R e^{2}\right)$, we must recognize that we have a singular asymptotic expansion. Near the particle we have the field governed by the steady Stokes equations (B 3). Far from the particle we have, most generally, the unsteady Oseen equations (B 8). (Note that the unsteady Oseen flow will asymptote to (B 9) or (B 10) at long times, $R e S l \ll R e^{2}$.) Under these conditions, to construct a uniformly valid velocity field, one must add these two velocity fields and subtract their common parts. Since the unsteady Oseen equation is point-forced, the associated velocity field will reduce to the point-forced Stokes field, or Stokeslet, as the particle centre is approached. Similarly, the inner Stokes field will reduce to the same Stokeslet at large distances from the particle. It is this Stokeslet that is common to both the inner and outer fields in their region of overlap, when $|\boldsymbol{r}|=O\left(a R e^{-1}\right)$. This Stokeslet field is given by the solution to

$$
-\nabla p_{0}^{p}+\nabla^{2} \boldsymbol{u}_{0}^{p}=\boldsymbol{F}_{s}^{H}(t) \delta(\boldsymbol{r}), \quad \nabla \cdot \boldsymbol{u}_{0}^{p}=0 .
$$

Thus, the uniformly valid velocity field for long timescales is given by

$$
u_{l}^{u v}=u_{0}+v_{0}^{p+}-u_{0}^{p}
$$

where $\boldsymbol{u}_{0}$ and $\boldsymbol{v}_{0}^{p+}$ are given by solutions to (B 3) and (B 8), respectively.

Now, to construct a velocity field valid for all time, we must consider the two velocity fields, (B12) and (B14), which are uniformly valid in space albeit for different timescales, and determine those features which are common to both. For long times (i.e. $\left.R e S l=R e^{2}\right), v_{0}$ will reduce to $\left(u_{0}+v_{0}^{p}-u_{0}^{p}\right)$, where $\left(v_{0}^{p}-u_{0}^{p}\right)$ represents the singular perturbation to Stokes flow from weak unsteadiness. Recall that $v_{0}^{p}$ is given by the solution to point-forced unsteady Stokes equations (B 4). For short times (i.e. $\left.R e S l \gg R e^{2}\right), u_{l}^{u v}$ will reduce to $\left(u_{0}+v_{0}^{p}-u_{0}^{p}\right)$ to leading order as well. This is because the convective term in the governing equation for $v_{0}^{p+},(\mathrm{B} 8)$, is only a small correction that would not modify the $v_{0}^{p}$-field to leading order anywhere in the fluid domain, as discussed in $\S \mathrm{B} .1$ above. Thus, $v_{0}^{p+}$ reduces to $v_{0}^{p}$ under the short timescales corresponding to this condition.

In addition, there is another portion of $v_{0}^{p+}$ that is also common to $R e v_{1}$. That is, the point-forced regular perturbation field, $\operatorname{Rev}_{1}^{p}$, given by

$$
-\nabla \Pi_{1}^{p}+\nabla^{2} \boldsymbol{v}_{1}^{p}-\operatorname{ReSl} \frac{\partial \boldsymbol{v}_{1}^{p}}{\partial t}=-U_{s}(t) \cdot \nabla v_{0}^{p}, \quad \nabla \cdot v_{1}^{p}=0
$$

Recall that the superscript + means that convective terms are retained at leading order. Thus, the uniformly valid velocity field valid for all time scales is given by

$$
\begin{aligned}
\boldsymbol{u}^{u v} & =\boldsymbol{u}_{s}^{u v}+\boldsymbol{u}_{l}^{u v}-\left(\boldsymbol{u}_{0}+\boldsymbol{v}_{0}^{p}-\boldsymbol{u}_{0}^{p}\right)-\operatorname{Re} \boldsymbol{v}_{1}^{p} \\
& =\boldsymbol{v}_{0}+\operatorname{Re} \boldsymbol{v}_{1}+\operatorname{Rev}_{1}^{p+}-\operatorname{Re} \boldsymbol{v}_{1}^{p}
\end{aligned}
$$

where

$$
\operatorname{Rev}_{1}^{p+}=\boldsymbol{v}_{0}^{p+}-\boldsymbol{v}_{0}^{p}
$$


which is given by the solution to

$$
\begin{gathered}
-\nabla \Pi_{1}^{p+}+\nabla^{2} v_{1}^{p+}-\operatorname{ReSl} \frac{\partial v_{1}^{p+}}{\partial t}+\operatorname{Re} U_{s}(t) \cdot \nabla v_{1}^{p+}=-U_{s}(t) \cdot \nabla v_{0}^{p} \\
\nabla \cdot v_{1}^{p+}=0 .
\end{gathered}
$$

\section{Appendix C}

In this section we solve for the Fourier transformed outer velocity field, $\widehat{\boldsymbol{v}_{1}^{p+}}$, and the resulting quantity, $f_{\text {out }}\left(\widehat{\operatorname{Re}}_{1}^{p+}\right)$. We begin by taking the Fourier transform of (B 4a):

$$
-2 \pi \mathrm{i} k \widehat{\Pi}_{0}^{p}-4 \pi^{2} k^{2} \widehat{\boldsymbol{v}_{0}^{p}}=\operatorname{ReSl} \frac{\partial \widehat{\boldsymbol{v}_{0}^{p}}}{\partial t}+\boldsymbol{F}_{s}^{H}(t)
$$

If we then make use of the continuity equation, which takes the form in Fourier space

$$
\boldsymbol{k} \cdot \widehat{\boldsymbol{v}_{0}^{p}}=0
$$

one finds the Fourier transformed pressure by taking the dot product of (C1) with $k$ :

$$
\widehat{\Pi_{0}^{p}}=\frac{\boldsymbol{k} \cdot \boldsymbol{F}_{s}^{H}(t)}{-2 \pi \mathrm{i} k^{2}}
$$

Combining this with (C1) and rearranging we have

$$
\frac{\partial \widehat{v}_{0}^{p}}{\partial t}+\frac{4 \pi^{2} k^{2}}{R e S l} \widehat{v_{0}^{p}}=\frac{F_{s}^{H}(t)}{R e S l} \cdot(n n-l),
$$

where $\boldsymbol{n}=\boldsymbol{k} / \boldsymbol{k}$. The solution to this equation is then our solution for $\widehat{\boldsymbol{v}_{0}^{p}}$ :

$$
\widehat{\boldsymbol{v}_{0}^{p}}=\int_{-\infty}^{t} \frac{\boldsymbol{F}_{s}^{H}(s)}{\operatorname{ReSl}} \mathrm{e}^{-4 \pi^{2} k^{2}(t-s) / R e S l} \mathrm{~d} s \cdot(\boldsymbol{n} \boldsymbol{n}-\boldsymbol{\eta}) .
$$

Next we take the Fourier transform of (B 18a) and obtain

$$
-2 \pi \mathrm{i} k \widehat{\Pi_{1}^{p+}}-4 \pi^{2} k^{2} \widehat{v_{1}^{p+}}=\operatorname{ReSl} \frac{\partial \widehat{v_{1}^{p+}}}{\partial t}-2 \pi \mathrm{i} \operatorname{Re} \boldsymbol{U}_{s}(t) \cdot \widehat{k} \widehat{v_{1}^{p+}}-2 \pi \mathrm{i} U_{s}(t) \cdot \boldsymbol{k} \widehat{v_{0}^{p}} .
$$

From the application of the continuity equation we find

$$
\widehat{\Pi_{1}^{p+}}=0 \text {. }
$$

Now (C6) takes the form

$$
\frac{\partial \widehat{v_{1}^{p+}}}{\partial t}+\left(\frac{4 \pi^{2} k^{2}-2 \pi \mathrm{i} R e U_{s}(t) \cdot \boldsymbol{k}}{\operatorname{ReSl}}\right) \widehat{\boldsymbol{v}_{1}^{p+}}=\frac{2 \pi \mathrm{i} U_{s}(t) \cdot \boldsymbol{k}}{\operatorname{ReSl}} \widehat{\boldsymbol{v}_{0}^{p}},
$$

and performing the integration and substituting for $\widehat{v_{0}^{p}}$ from (C 5), we obtain

$$
\begin{aligned}
& \widehat{\boldsymbol{v}_{1}^{p+}}=\left[\int_{-\infty}^{t} \frac{2 \pi \mathrm{i} U_{s}(s) \cdot \boldsymbol{k}}{\operatorname{ReS} S}\left(\int_{-\infty}^{s} \frac{\boldsymbol{F}_{s}^{H}(q)}{\operatorname{Re} S l} e^{-4 \pi^{2} k^{2}(t-q) / R e S l} \mathrm{~d} q\right)\right. \\
& \left.\times \mathrm{e}^{2 \pi \mathrm{iRe}\left(\boldsymbol{Y}_{s}(t)-\boldsymbol{Y}_{s}(s)\right) \cdot k / R e s l} \mathrm{~d} s\right] \cdot(\boldsymbol{n n}-\boldsymbol{l}) .
\end{aligned}
$$


Now we can compute

$$
\left.f_{\text {out }}(\widehat{\operatorname{Rev}})_{1}^{p+}\right)=\operatorname{Re}\left(\operatorname{ReSl} \frac{\partial \widehat{\boldsymbol{v}_{1}^{p+}}}{\partial t}-2 \pi \mathrm{i} \operatorname{Re} U_{s}(t) \cdot \boldsymbol{k} \widehat{\boldsymbol{v}_{1}^{p^{+}}}\right),
$$

to find

$$
\begin{aligned}
& \boldsymbol{f}_{\text {out }}\left(\widehat{\left.\operatorname{Re} v_{1}^{p+}\right)}=2 \pi \mathrm{i} \operatorname{Re} U_{s}(t) \cdot \boldsymbol{k} \int_{-\infty}^{t} \frac{\boldsymbol{F}_{s}^{H}(s)}{\operatorname{ReSl}} \mathrm{e}^{-4 \pi^{2} k^{2}(t-s) / \operatorname{Re} S t} \mathrm{~d} s \cdot(\boldsymbol{n n}-\boldsymbol{l})\right.
\end{aligned}
$$

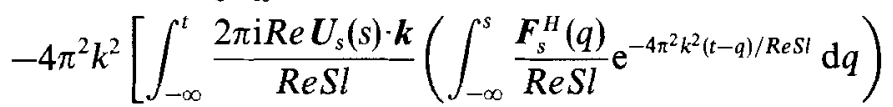

$$
\begin{aligned}
& \left.\times \mathrm{e}^{2 \pi \mathrm{iRe}\left(\boldsymbol{Y}_{s}(t)-\boldsymbol{Y}_{s}(s)\right) \cdot \boldsymbol{k} / \operatorname{ReSl}} \mathrm{d} s\right] \cdot(\boldsymbol{n n}-\boldsymbol{I}) .
\end{aligned}
$$

Next we note that by performing an integration by parts we have

$$
\begin{aligned}
\int_{-\infty}^{s} \frac{F_{s}^{H}(q)}{\operatorname{ReSl}} \mathrm{e}^{-4 \pi^{2} k^{2}(t-q) / R e S l} \mathrm{~d} q= & \frac{F_{s}^{H}(s)}{4 \pi^{2} k^{2}} e^{-4 \pi^{2} k^{2}(t-s) / R e S l} \\
& -\int_{-\infty}^{s} \frac{\dot{F}_{s}^{H}(q)}{4 \pi^{2} k^{2}} \mathrm{e}^{-4 \pi^{2} k^{2}(t-q) / R e S l} \mathrm{~d} q .
\end{aligned}
$$

Thus combining (C 12) with (C 11) we have finally

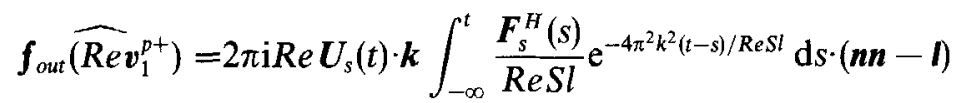

$$
\begin{aligned}
& -\int_{-\infty}^{t} \frac{2 \pi \mathrm{i} R e \boldsymbol{U}_{s}(s) \cdot \boldsymbol{k}}{\operatorname{ReSl}} \boldsymbol{F}_{s}^{H}(s) \mathrm{e}^{-4 \pi^{2} k^{2}(t-s) / R e S l} \\
& \times \mathrm{e}^{2 \pi \mathrm{iRe}\left(\boldsymbol{Y}_{s}(t)-\boldsymbol{Y}_{s}(s)\right) \cdot k / R e S l} \mathrm{~d} \cdot(\boldsymbol{n n}-\boldsymbol{l}) \\
& +\int_{-\infty}^{t} \int_{-\infty}^{s} \frac{2 \pi \mathrm{i} \operatorname{Re} \boldsymbol{U}_{s}(s) \cdot \boldsymbol{k}}{\operatorname{ReS} l} \mathrm{e}^{2 \pi \mathrm{iRe}\left(\boldsymbol{Y}_{s}(t)-\boldsymbol{Y}_{s}(s)\right) \cdot k / \operatorname{Resl}} \dot{\boldsymbol{F}}_{s}^{H}(q) \\
& \times \mathrm{e}^{-4 \pi^{2} k^{2}(t-q) / R e s l} \mathrm{~d} q \mathrm{~d} s \cdot(\boldsymbol{n} \boldsymbol{n}-\boldsymbol{l}) .
\end{aligned}
$$

If we change the order of integration in the last integral of $(\mathrm{C} 13)$ and integrate with respect to $s$, we obtain the result in (6.5) in the main text.

\section{Appendix D. The approach to steady state in Oseen flows}

$$
\text { By E.J. Hinch }
$$

Department of Applied Mathematics and Theoretical Physics, University of Cambridge, Silver Street, Cambridge CB3 9EW, UK

In $\$ 6$, Lovalenti \& Brady found an interesting variety of approaches to the steady state in Oseen flows, like $t^{-2}, t^{-1}, t^{-2} \mathrm{e}^{-t}$ and $t^{-5 / 2} \mathrm{e}^{-t}$. The purpose of this appendix is to describe the different mechanisms responsible for this variety and thus to give a simple derivation of each of these behaviours. The arguments are given in terms of dimensional variables, and they apply only far from the particle on the Oseen lengthscale $v / U$ and only at long times when $U t>v / U$.

Steady Oseen wake. First we need to understand the steady uniform Oseen flow $U$ past a fixed sphere of radius $a$ at the origin. Vorticity generated at the sphere 
is advected downstream, say in the $z$-direction, confined to a wake which grows diffusively across the flow to $\delta(z)=(v z / U)^{\frac{1}{2}}$, where $z / U$ is the time taken to travel at speed $U$ from the particle to position $z$. The wake has a momentum deficit $\int \rho U(U-u) \mathrm{d} x \mathrm{~d} y$ equal to the $\mathrm{drag} 6 \pi \mu a U$. Note that for the Oseen equation the momentum flux is $U u$ rather than $u^{2}$. In the wake the velocity disturbance decays therefore like $6 \pi \mu a U / \rho U \pi \delta^{2}=6 U a / z$, more precisely with a Gaussian spread

$$
U-u=\frac{6 \pi \mu a}{\rho} \frac{\mathrm{e}^{-\left(x^{2}+y^{2}\right) /(4 v z / U)}}{4 \pi v z / U}=\frac{3 U a}{2 z} \mathrm{e}^{-U\left(x^{2}+y^{2}\right) / 4 v z} .
$$

Now the momentum deficit in the wake means that there is also a mass deficit $\int \rho(U-u) \mathrm{d} x \mathrm{~d} y=6 \pi \mu a$. The corresponding mass supplied upstream is dispersed by the particle acting as a spherically symmetric irrotational point mass source, with a radial potential flow $u_{r}=6 \pi \mu a / \rho 4 \pi r^{2}$. (See the streamfunction for the Oseen flow with wake and source flow, e.g. Lamb 1932 p. 612.)

Start-up from rest. For start-up, there will be a wake downstream only to $z=U t$. This has a mass-flow deficit in $0<z<U t$. Hence there must be a mass sink of strength $6 \pi \mu a$ in the region of the end of the wake near $z=U t$. This will induce a flow enhancement at the particle, distance $U t$, of $6 \pi \mu a / \rho 4 \pi(U t)^{2}$ and so an additional force on the particle

$$
6 \pi \mu a \frac{3 a v}{2(U t)^{2}}=6 \pi \mu a U \frac{U a}{v} \frac{3}{32}\left(\frac{4 v}{U^{2} t}\right)^{2},
$$

i.e. the non-dimensional extra force $\frac{3}{8} \frac{1}{4} t^{-2}$ of equation $(6.21 a)$.

Stopping. For stopping, there is a downstream wake which will diffuse cylindrically away with disturbance velocities on the $z$-axis $6 \pi \mu a / \rho 4 \pi v t$. At any point on the axis in the wake, half of this velocity disturbance comes from sources of velocity disturbance downstream of the point and half from sources upstream. But the particle has no upstream wake, so it feels just half of this. Hence there is a force opposed to the original flow

$$
-6 \pi \mu a \frac{3 a}{4 t}=-6 \pi \mu a U \frac{U a}{v} \frac{3}{16}\left(\frac{4 v}{U^{2} t}\right),
$$

i.e. the non-dimensional extra force $-\frac{3}{16} t^{-1}$ of equation (6.23a).

Step increase. For a step increase from $U_{o}$ to $U_{n}$, the old and new wakes will join at $z=U_{n} t$. They will have the same mass deficit $6 \pi \mu a$, the same width $(v t)^{\frac{1}{2}}$ with $t$ the time since passing the particle, but different false origins. Because the mass deficit is the same, there is no need for any irrotational source/sink motion as in the case of starting from rest. Note that the mass deficit is independent of the velocity, so long as the velocity is non-zero. This is the reason for the significant difference between starting from rest and increasing from a small non-zero velocity. With no irrotational flow effect, the influence of the join in the wakes comes from vorticity diffusing back upstream to the particle, and this is exponentially small.

We calculate the velocity disturbance at the particle due to the change in the wakes by considering the wake to be due to an advected line distribution of diffusing momentum sources. Now the fundamental solution of the unsteady Stokes equation, the governing equation in the advected frame, for a point source of momentum $\boldsymbol{F}$ at 
the origin at the initial instant is

$$
\boldsymbol{u}=\boldsymbol{F} \frac{\mathrm{e}^{-r^{2} / 4 v t}}{(4 \pi v t)^{\frac{3}{2}}}+\nabla \phi \quad \text { with pressure } p=-\rho \frac{\partial \phi}{\partial t},
$$

i.e. a diffusing momentum source plus a potential flow correction in order to satisfy the incompressibility condition. Clearly the potential is a first harmonic $\phi=(\boldsymbol{F} \cdot \boldsymbol{x}) f(r, t)$, and it is easy to find from the incompressibility condition

$$
f=-\frac{1}{\rho 4 \pi r^{3}}-\frac{1}{6 \rho v t(4 \pi v t)^{\frac{3}{2}}} \int_{r}^{\infty}\left(\xi-r^{-3} \xi^{4}\right) \mathrm{e}^{-\xi^{2} / 4 v t} \mathrm{~d} \xi
$$

This solution can also be obtained from the Fourier transform in equation (C5). In the direction of $\boldsymbol{F}$, we thus have a flow in the far field

$$
u \sim \frac{F}{\rho}\left[\frac{1}{2 \pi r^{3}}-\frac{4 v t r^{-2}}{(4 \pi v t)^{\frac{3}{2}}} \mathrm{e}^{-r^{2} / 4 v t}\right] .
$$

The first term is a potential dipole flow, and the second is due to the little vorticity which has diffused a distance $r$.

Now the old wake is due to momentum sources $6 \pi \mu a$ per unit length along the $z$-axis in $z>U_{n} t$ with ages, the time lapsed since the particle was at the location, $t_{o}(z)=t+\left(z-U_{n} t\right) / U_{o}$. These will be replaced by sources of the same strength on the new wake but with ages $t_{n}(z)=t+\left(z-U_{n} t\right) / U_{n}$. As the source strengths are equal, the potential dipole flow terms cancel precisely, leaving a velocity disturbance at the particle from the exponentially small diffusion of vorticity

$$
\int_{U_{n} t}^{\infty} \frac{6 \pi \mu a}{\rho}\left(\frac{\mathrm{e}^{-z^{2} / 4 v t_{o}(z)}}{z^{2} \pi\left(4 \pi v t_{o}(z)\right)^{\frac{1}{2}}}-\frac{\mathrm{e}^{-z^{2} / 4 v t_{n}(z)}}{z^{2} \pi\left(4 \pi v t_{n}(z)\right)^{\frac{1}{2}}}\right) \mathrm{d} z .
$$

There is an interesting competition in the exponential exponents above, between the decay from the spatial variation like $-z^{2}$ and the growth from the spreading with age $1 / t(z)$. The first exponent is

$$
-\frac{z^{2}}{4 v t_{o}(z)}=-\frac{U_{n}^{2} t^{2}\left(1+\zeta / U_{n} t\right)^{2}}{4 v t\left(1+\zeta / U_{o} t\right)} \text { with } \zeta=z-U_{n} t
$$

Thus if $2 / U_{n} t>1 / U_{o} t$, i.e. $U_{o}>\frac{1}{2} U_{n}$, then the spatial decay in the numerator wins, while if $U_{o}<\frac{1}{2} U_{n}$ then the age effect in the denominator wins.

Case of a small step increase $U_{o}>\frac{1}{2} U_{n}$, i.e. the case of the spatial decay winning. In this case the major contribution to the integral comes from within $O(v / U)$ of $z=U_{n} t$. Expanding the exponents about the join of the wakes at $z=U_{n} t$

$-\frac{U_{n}^{2} t^{2}}{4 v t}\left[1+\left(\frac{2}{U_{n} t}-\frac{1}{U_{o} t}\right)\left(z-U_{n} t\right)+\ldots\right]$ and $-\frac{U_{n}^{2} t^{2}}{4 v t}\left[1+\frac{1}{U_{n} t}\left(z-U_{n} t\right)+\ldots\right]$.

The integral for the velocity disturbance can be evaluated

$$
\frac{6 \pi \mu a}{\rho} \frac{\mathrm{e}^{-U_{n}^{2} t / 4 v}}{U_{n}^{2} t^{2} \pi(4 \pi v t)^{\frac{1}{2}}} \frac{4 v\left(U_{n}-U_{o}\right)}{U_{n}\left(2 U_{o}-U_{n}\right)} .
$$

This velocity will produce the non-dimensional extra force

$$
\frac{3}{8 \pi^{\frac{1}{2}}} \frac{1-b}{2 b-1} t^{-\frac{5}{2}} \mathrm{e}^{-t}
$$

of equation $(6.21 d)$. 
This result for a small step increase can be applied to the case of a step decrease to a non-zero value, producing the non-dimensional reduction of force

$$
\frac{3}{8 \pi^{\frac{1}{2}}} \frac{1-b}{2-b} t^{-\frac{5}{2}} \mathrm{e}^{-b^{2} t}
$$

of equation $(6.23 b)$.

Case of a large step increase $U_{o}<\frac{1}{2} U_{n}$, i.e. the case of the ageing winning. In this case the major contribution comes from where the first exponent is a maximum, which occurs at $z=2\left(U_{n}-U_{o}\right) t\left(>U_{n} t\right.$ if $\left.U_{o}<\frac{1}{2} U_{n}\right)$ where $t_{o}(z)=\left(U_{n} / U_{o}-1\right) t$. Near its maximum, the first exponent is

$$
-\frac{z^{2} U_{o}}{4 v\left(z+\left(U_{o}-U_{n}\right) t\right)}=-\frac{U_{o}\left(U_{n}-U_{o}\right) t}{v}-\frac{U_{o}\left(z-2\left(U_{n}-U_{o}\right) t\right)^{2}}{4 v\left(U_{n}-U_{o}\right) t}+\ldots
$$

Integrating this Gaussian in $z$ gives a velocity disturbance

$$
\frac{6 \pi \mu a}{\rho} \frac{\mathrm{e}^{-U_{o}\left(U_{n}-U_{o}\right) t / v}}{4\left(U_{n}-U_{o}\right)^{2} t^{2} \pi}
$$

This will produce the non-dimensional extra force

$$
\frac{3}{32} \frac{1}{(1-b)^{2}} t^{-2} \mathrm{e}^{-4 b(1-b) t}
$$

of equation $(6.21 b)$.

In the marginal case $U_{o}=\frac{1}{2} U_{n}$, the spatial decay and ageing effect balance, with the first exponent having a maximum at $z=U_{n} t$. Thus we have just half the above Gaussian, and so precisely half its answer, i.e. a non-dimensional force $\frac{3}{16} t^{-2} \mathrm{e}^{-t}$ of equation $(6.21 \mathrm{c})$.

\section{REFERENCES}

Adrian, R. J. 1991 Particle-imaging techniques for experimental fluid mechanics. Ann. Rev. Fluid Mech. 23, 261-304.

BASSET, A. B. 1888 A Treatise on Hydrodynamics, vol. 2. Cambridge: Deighton Bell.

BENTwich, M. \& MiLOH, V. 1978 The unsteady matched Stokes-Oseen solution for the flow past a sphere. J. Fluid Mech. 88, 17-32.

BRENNER, H. 1961 The Oseen resistance of a particle of arbitrary shape. J. Fluid Mech. 11, 604-610.

BRENNER, H. \& COX, R.G. 1963 The resistance to a particle of arbitrary shape in translational motion at small Reynolds numbers. J. Fluid Mech. 17, 561-595.

ChILDRESS, S. 1964 The slow motion of a sphere in a rotating, viscous fluid. J.Fluid Mech. 20, 305-314.

Cox, R.G. 1965 The steady motion of a particle of arbitrary shape at small Reynolds numbers. J. Fluid Mech. 23, 625-643.

Cox, R.G. \& BRENNER, H. 1968 The lateral migration of solid particles in Poiseuille flow - I. Theory. Chem. Engng Sci. 23, 147-173.

GAvzE, E. 1990 The accelerated motion of rigid bodies in non-steady Stokes flow. Intl J. Multiphase Flow 16, 153-166.

HAPPEL, J. \& BRENNER, H. 1986 Low Reynolds Number Hydrodynamics. Martinus-Nijhoff.

Ho, B.P. \& LeAL, L. G. 1974 Inertial migration of rigid spheres in two-dimensional unidirectional flows. J. Fluid Mech. 65, 365-400.

LAMB, H. 1932 Hydrodynamics, 6th edn. Cambridge University Press.

LAWRENCE, C. J. \& WeinBauM, S. 1986 The force on an axisymmetric body in linearized, timedependent motion: a new memory term. J.Fluid Mech. 171, 209-218. 
LAWrenCE, C. J. \& WeInBaum, S. 1988 The unsteady force on a body at low Reynolds number; the axisymmetric motion of a spheroid. J. Fluid Mech. 189, 463-489.

LEAL, L.G. 1980 Particle motions in a viscous fluid. Ann. Rev. Fluid Mech. 12, 435-476.

LOVALENTI, P. M. \& BRADY, J. F. $1993 a$ The force on a sphere in a uniform flow with small-amplitude oscillations at finite Reynolds number. J. Fluid Mech. 256, 607-614.

LOVAlENTI, P.M. \& BRADY, J.F. $1993 b$ The force on a bubble, drop, or particle in arbitrary time-dependent motion at small Reynolds number. Phys. Fluids A 5, 2104-2116.

MAXEY, M.R. \& RileY, J.J. 1983 Equation of motion for a small rigid sphere in a nonuniform flow. Phys. Fluids 26, 883-889.

MAXWORTHY, T. 1965 Accurate measurements of sphere drag at low Reynolds numbers. J.Fluid Mech. 23, 369-372.

MeI, R. \& AdRIAN, R. J. 1992 Flow past a sphere with an oscillation in the free-stream velocity and unsteady drag at finite Reynolds number. J. Fluid Mech. 237, 323-341.

Mei, R., Adrian, R.J. \& Hanratty, T. J. 1991 Particle dispersion in isotropic turbulence under Stokes drag and Basset force with gravitational settling. J. Fluid Mech. 225, 481-495.

Mei, R., LaWrence, C.J. \& Adrian, R.J. 1991 Unsteady drag on a sphere at finite Reynolds number with small fluctuations in the free-stream velocity. J. Fluid Mech. 233, 613-631.

OCKENDON, J. R. 1968 The unsteady motion of a small sphere in a viscous liquid. J. Fluid Mech. 34, 229-239.

OSEEN, C.W. 1910 Über die Stokes'sche Formel und über eine verwandte Aufgabe in der Hydrodynamik. Ark. f. Mat. Astr. och Fys. 6 no.29.

OseEn, C.W. 1913 Über den Gültigkeitsbereich der Stokesschen Widerstandsformel. Ark. f. Mat. Astr. och Fys. 9 no.16.

PozRIKIDIs, C. 1989 A study of linearized oscillatory flow past particles by the boundary-integral method. J. Fluid Mech. 202, 17-41.

Proudman, I. \& Pearson, J.R.A. 1957 Expansion at small Reynolds numbers for the flow past a sphere and a circular cylinder. J. Fluid Mech. 2, 237-262.

Reeks, M.W. \& McKeE, S. 1984 The dispersive effects of Basset history forces on the particle motion in a turbulent flow. Phys. Fluids 27, 1573-1582.

Rubinow, S. I. \& KelleR, J. B. 1961 The transverse force on a spinning sphere moving in a viscous fluid. J. Fluid Mech. 11, 447-459.

SAFFMAN, P.G. 1965 The lift on a small sphere in a slow shear flow. J. Fluid Mech. 22, 385-400.

SANO, T. 1981 Unsteady flow past a sphere at low Reynolds number. J. Fluid Mech. 112, 433-441.

STONE, H. A. \& BRADY, J. F. 1993 Inertial effects on the rheology of a suspension and on the motion of isolated rigid particles. J. Fluid Mech. (to be submitted).

Williams, W. E. 1966 A note on the slow vibrations in a viscous fluid. J. Fluid Mech. 25, 589-590.

YounG, J. B. \& HanRaTTY, T. J. 1991 Optical studies on the turbulent motion of solid particles in pipe flow. J. Fluid Mech. 231, 665-688. 